\title{
The rheology of systems containing rigid spheres suspended in both viscous and viscoelastic media, studied by Stokesian dynamics simulations
}

Citation for published version (APA):

Schaink, H. M., Slot, J. J. M., Jongschaap, R. J. J., \& Mellema, J. (2000). The rheology of systems containing rigid spheres suspended in both viscous and viscoelastic media, studied by Stokesian dynamics simulations. Journal of Rheology, 44(3), 473-498. https://doi.org/10.1122/1.551097

DOI:

$10.1122 / 1.551097$

Document status and date:

Published: 01/01/2000

Document Version:

Publisher's PDF, also known as Version of Record (includes final page, issue and volume numbers)

Please check the document version of this publication:

- A submitted manuscript is the version of the article upon submission and before peer-review. There can be important differences between the submitted version and the official published version of record. People interested in the research are advised to contact the author for the final version of the publication, or visit the $\mathrm{DOI}$ to the publisher's website.

- The final author version and the galley proof are versions of the publication after peer review.

- The final published version features the final layout of the paper including the volume, issue and page numbers.

Link to publication

\footnotetext{
General rights

- You may freely distribute the URL identifying the publication in the public portal. follow below link for the End User Agreement:

www.tue.nl/taverne

\section{Take down policy}

If you believe that this document breaches copyright please contact us at:

openaccess@tue.nl

providing details and we will investigate your claim.
}

Copyright and moral rights for the publications made accessible in the public portal are retained by the authors and/or other copyright owners and it is a condition of accessing publications that users recognise and abide by the legal requirements associated with these rights.

- Users may download and print one copy of any publication from the public portal for the purpose of private study or research.

- You may not further distribute the material or use it for any profit-making activity or commercial gain

If the publication is distributed under the terms of Article $25 \mathrm{fa}$ of the Dutch Copyright Act, indicated by the "Taverne" license above, please 


\title{
The rheology of systems containing rigid spheres suspended in both viscous and viscoelastic media, studied by Stokesian dynamics simulations
}

\author{
H. M. Schaink ${ }^{\text {a) }}$ \\ J. M. Burgers Centre, Rheology Group, Faculty of Applied Physics, \\ University of Twente, P.O. Box 217, 7500 AE Enschede, The Netherlands \\ J. J. M. Slot \\ Department for Physical, Analytical and Computational Chemistry, \\ DSM Research, P.O. Box 18, 6160 MD Geleen, The Netherlands \\ and J. M. Burgers Centre, Rheology Group, Faculty of Applied Physics, \\ University of Twente, P.O. Box 217, 7500 AE Enschede, The Netherlands \\ R. J. J. Jongschaap and J. Mellema \\ J. M. Burgers Centre, Rheology Group, Faculty of Applied Physics, \\ University of Twente, P.O. Box 217, 7500 AE Enschede, The Netherlands
}

(Received 24 May 1999; final revision received 27 January 2000)

\begin{abstract}
Synopsis
An extensive Stokesian dynamics study of the rheological behavior of suspensions of rigid spheres subjected to an oscillating shear strain is presented. Two types of suspensions are considered: (1) rigid spheres in a viscous medium, and (2) rigid spheres in a viscoelastic medium. For this latter system we need to extend the Stokesian dynamics method, which was originally developed by Brady and Bossis for particles suspended in a viscous medium. The derivation of the necessary equations for these extended Stokesian dynamics simulations is given. In this derivation we use the well known correspondence principle and apply it to the set of equations for the hydrodynamic forces and stresslets that are valid for spheres suspended in a viscous medium. Then, using Fourier transformation we obtain differential equations for these forces and stresslets in the viscoelastic case. The contribution of the Brownian motion of the spheres to the bulk stress is found to be independent of the viscoelastic properties of the suspending medium. As an example of a viscoelastic medium we have chosen the Maxwell fluid. In our computer simulations we have calculated both elastic and viscous moduli and compared these results with experimental data. For the case of spheres suspended in a viscous medium we find that the elastic modulus reaches a plateau at high frequencies. Finally we present a simple analytical model which accurately reproduces the hydrodynamic contribution to the viscosity of spheres suspended in a Maxwell medium. This model is used to interpret the experimental results of Aral and Kaylon [Aral, B. K. and D. M. Kaylon, J. Rheol. 41, 599-620 (1977)] that were obtained at Peclet numbers currently inaccessible to Stokesian dynamics simulations. (C) 2000 The Society of Rheology. [S0148-6055(00)00803-8]
\end{abstract}

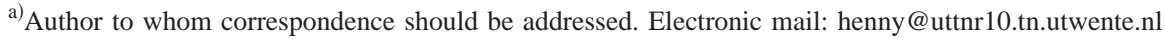




\section{INTRODUCTION}

Knowledge of the rheological properties of composite materials is clearly of great technological importance. In order to obtain an understanding of these properties, a systematic study is required. The simplest model system in which to study such properties is a suspension of rigid spheres in a viscous fluid. The observation of van der Werff et al. (1989) that this suspension displays viscoelastic behavior has triggered a considerable amount of experimental [Woutersen et al. (1994); Shikata and Pearson (1994)] and theoretical work [Brady (1993); Verberg et al. (1997)]. The current status regarding computer simulations of the rheology of suspensions and/or dispersions in viscous media is the following. Heyes and Mitchell (1994) have done Brownian dynamics simulations of spheres interacting through various soft potentials subjected to an oscillating shear strain. For the frequency dependence of the viscosity they obtained qualitative agreement with the experiments of van der Werff et al. (1989). However, in these simulations the hydrodynamic interactions between the spheres were only accounted for in an ad hoc manner. These hydrodynamic interactions are treated correctly in the Stokesian dynamics simulations done by Phung et al. (1996) and in the simulations of Ladd (1990). Both Phung and Ladd have studied dispersions subjected to simple shear and found quantitative agreement with experiments. Up to now Toivakka et al. (1995) have published the only report about simulations of suspensions subjected to an oscillating shear strain using an approximative Stokesian dynamics algorithm. However, due to the geometry of their system, a monolayer of spherical particles between two planar hard walls, it is not possible to compare their findings to the experimental results of three-dimensional suspensions. Finally, there are some simulations of dispersions of spheres in a viscous matrix using the lattice Boltzmann technique [Ladd (1994)] or the dissipative particle dynamics method [Koelman and Hoogerbrugge (1993); Boek et al. (1997)].

There is a huge amount of experimental data on the rheology of particle filled polymer melts. However, most of the reports that cover this subject deal with systems which contain polydisperse particles of irregular shape. Suspensions of well defined rigid spheres in viscoelastic media are less well studied. A few rheological experiments on glass beads in a viscoelastic matrix have been reported [Chan and Powell (1984); Ohl and Gleissle (1992); Aral and Kalyon (1997)]. Up to now computer simulations of this type of system have focused on the dynamics of a single sphere in various types of viscoelastic fluids. These calculations were done mainly with finite element techniques. Recently Feng et al. (1996) and Huang et al. (1998) have extended these techniques to calculate the sedimentation of many spheres in an Oldroyd-B fluid. In these finite element calculations the Brownian motion of the spheres is neglected. Another option is the development of lattice Boltzmann techniques which are able to model viscoelastic fluids [Giraud et al. (1998)]. This scheme has recently been applied to calculate the motion of a Newtonian drop in a viscoelastic fluid [Wagner et al. (1999)]. To date this new lattice Boltzmann scheme has not yet been used in simulations of suspensions of spheres.

The first attempt to construct a Stokesian dynamics scheme for spheres in a nonNewtonian medium was made by Phillips (1996). Using a perturbation scheme he derived expressions for the hydrodynamic force exerted on the particles by a second order fluid. As a result, his approach is only valid for fluids for which the relaxation time is short relative to the time scale of the (slow) fluid flow. Long time memory effects cannot be studied using this scheme. It is not clear in his paper how he treats the Brownian force exerted on the particles.

In this article we present a comparative Stokesian dynamics [Brady and Bossis (1988)] study of two types of suspensions subjected to an oscillating shear strain: (1) rigid 
spheres suspended in a viscous medium, and (2) rigid spheres suspended in a viscoelastic medium. For the rigid spheres suspended in a viscoelastic medium we derive expressions for hydrodynamic force as well as for Brownian force using the fluctuation-dissipation theorem. In this article we will restrict ourselves to overall linear viscoelastic response. One of the simplest models for such linear viscoelastic behavior is the Maxwell fluid. The reason why we restrict ourselves to linear viscoelasticity is that all fluids exhibit this behavior for sufficiently small deformations and deformation rates. This study is to be considered as a first step of a more challenging study of suspensions in media that exhibit nonlinear viscoelastic behavior.

This article is organized as follows. First we will give a resumé of the Stokesian dynamics simulation technique for spheres in a viscous medium subjected to an oscillating shear. Then we discuss how the equations governing the motion of the particles and the calculation of the bulk stress will change when the viscous medium is replaced by a Maxwell medium. We present simulation results for both types of systems and compare these results, where possible, with experimental data. We also present an analytic model for the calculation of the hydrodynamic contribution to the viscosity for spheres in a Maxwell medium.

\section{THEORY}

\section{A. The stress tensor}

We will be studying a system of $N$ spheres which are either suspended in a viscous fluid or in a Maxwell medium. For a viscous fluid the microscopic stress tensor $\sigma(\mathbf{r}, t)$ is given by

$$
\sigma(\mathbf{r}, t)=-p(\mathbf{r}, t) \mathbf{I}+\sigma^{\prime}(\mathbf{r}, t)=-p(\mathbf{r}, t) \mathbf{I}+2 \eta_{m} \mathbf{D}(r, t),
$$

where $p(\mathbf{r}, t)$ represents the local hydrostatic pressure, and $\eta_{m}$ is the viscosity. For a Maxwell medium with one relaxation time $\tau$ this $\sigma^{\prime}(\mathbf{r}, t)$ obeys the differential equation

$$
\tau \frac{d \sigma^{\prime}(\mathbf{r}, t)}{d t}+\sigma^{\prime}(\mathbf{r}, t)=2 \eta_{m} \mathbf{D}(\mathbf{r}, t),
$$

where $d / d t \equiv \partial / \partial t+v \cdot \nabla$ denotes the material time derivative. In Eqs. (1) and (2) we have used the microscopic rate-of-strain tensor, which is defined by

$$
\mathbf{D}(\mathbf{r}, t)=\frac{1}{2}\left\{\nabla \mathbf{v}(\mathbf{r}, t)+[\nabla \mathbf{v}(\mathbf{r}, t)]^{T}\right\},
$$

where $\mathbf{v}(\mathbf{r}, \mathbf{t})$ is the velocity field in the medium.

In order to study the rheology of such a suspension we need to be able to calculate the macroscopic stress tensor of a configuration of particles. This macroscopic stress tensor consists of a fluid (medium) contribution and a particle contribution,

$$
\mathbf{T}=\mathbf{T}_{m}+\mathbf{T}_{p} .
$$

The medium contribution is given by

$$
\mathbf{T}_{m}(t)=\frac{1}{V} \int_{V_{m}(t)} d^{3} r \sigma(\mathbf{r}, t) .
$$

Here $V_{m}(t)$ represents the volume occupied by the fluid at time $t$. For suspensions of rigid particles it was shown by Batchelor $(1970,1977)$ that the particle contribution to the 
bulk stress is given by a hydrodynamic contribution $\mathbf{T}_{H}$, which is the sum of all the stresslets $\mathbf{S}_{\alpha}$ of the individual particles, plus a contribution $\mathbf{T}_{B}$ due to interparticle interactions and Brownian forces,

$$
\mathbf{T}_{p} \equiv \mathbf{T}_{H}+\mathbf{T}_{B}=\frac{1}{V} \sum_{\alpha}^{N} \mathbf{S}_{\alpha}-\frac{1}{V} \sum_{\alpha}^{N} \mathbf{r}_{\alpha} \mathbf{f}_{\alpha}
$$

where $\mathbf{f}_{\alpha}$ is the total force due to the interactions on a single particle located at $\mathbf{r}_{\alpha}$. The stresslet $\mathbf{S}_{\alpha}$ depends on the local extra stress $\sigma^{\prime}$ in the ambient medium,

$$
\mathbf{S}_{\alpha}=\frac{1}{2} \int_{A_{\alpha}} d^{2} r\left(\left(\mathbf{r}-\mathbf{r}_{\alpha}\right)\left(\sigma^{\prime} \cdot \mathbf{n}\right)+\left(\sigma^{\prime} \cdot \mathbf{n}\right)\left(\mathbf{r}-\mathbf{r}_{\alpha}\right)-\frac{2}{3} \mathbf{I}\left[\left(\mathbf{r}-\mathbf{r}_{\alpha}\right) \cdot \sigma^{\prime} \cdot \mathbf{n}\right]\right),
$$

because the isotropic pressure does not contribute to the stresslet. The integral in this equation is over the surface $A_{\alpha}$ of the particle at $\mathbf{r}_{\alpha}, \mathbf{n}$ is a normal vector of this surface pointing into the fluid [Batchelor (1970)], and I is the unit tensor. In Secs. II B and II C we will show how the bulk stress, given by Eqs. (4)-(6), can be obtained in a Stokesian dynamics simulation.

\section{B. Stokesian dynamics of spheres in a viscous fluid}

Here we will recapitulate how Stokesian dynamics simulations of spheres in a viscous fluid are performed. We will only pay attention to those aspects of the algorithm that need to be modified in order to be able to simulate the rheology of spheres in a viscoelastic medium. This will be extended in Sec. III C. For more information about the Stokesian dynamics (SD) method we refer the reader to the work of Brady and Bossis $(1988,1989)$.

We will assume for simplicity that all $N$ spheres in the suspension have the same mass $m$ and the same radius $a$ and that their motion is described by the following $3 N$-dimensional Langevin equation:

$$
m \frac{d v}{d t}=f_{H}+f_{B}
$$

where $f_{H} \equiv\left(\mathbf{f}_{H, 1}, \mathbf{f}_{H, 2}, \ldots, \mathbf{f}_{H, N}\right)^{T}$ is a vector of the hydrodynamic forces on the particles with similar definitions for the $3 N$-dimensional velocity and Brownian force vectors $v$ and $f_{B}$. The hydrodynamic force vector $\mathbf{f}_{H, \alpha}(t)$ on particle $\alpha$ is defined by

$$
\mathbf{f}_{H, \alpha}(t)=\int_{A_{\alpha}} d^{2} r \sigma^{\prime}(\mathbf{r}, t) \cdot \mathbf{n}
$$

because here also there is no contribution from the isotropic pressure. The hydrodynamic force vector $f_{H}$ and the stresslet vector $S \equiv\left(\mathbf{S}_{1}, \mathbf{S}_{2}, \ldots, \mathbf{S}_{N}\right)^{T}$, with $\mathbf{S}_{\alpha}$ defined in Eq. (7), are linearly related to the particle velocity vector and the rate-of-strain tensor by the so called grand resistance matrix,

$$
\left(\begin{array}{c}
f_{H} \\
S
\end{array}\right)=\eta_{m}\left(\begin{array}{ll}
R_{f v} & R_{f D} \\
R_{S v} & R_{\mathrm{SD}}
\end{array}\right)\left(\begin{array}{c}
v^{\infty}-v \\
D^{\infty}
\end{array}\right)
$$

In this expression $R_{f v}$ is a resistance matrix that describes the coupling between the hydrodynamic forces acting on the particles and their relative velocities with respect to the velocity $v^{\infty}$ of the ambient flow field and $R_{f D}$ is a resistance matrix that describes the coupling between these forces and the ambient flow field rate-of-strain tensor $D^{\infty}$ 
$\equiv\left[\mathbf{D}^{\infty}\left(\mathbf{r}_{1}\right), \ldots, \mathbf{D}^{\infty}\left(\mathbf{r}_{N}\right)\right]^{T}$ evaluated at the particle positions. The resistance matrices $R_{S v}$ and $R_{\mathrm{SD}}$ are similarly defined. These resistance matrices only depend on the shape of the particles and their position and orientation in space [Brenner (1964)]. More information on the resistance matrices can be found in a book by Kim and Karilla (1991). Although the four quantities $R_{f v}, R_{f D}, R_{S v}$, and $R_{\mathrm{SD}}$ are refered to as resistance matrices, it is clear from Eq. (10) that they are not matrices in the strict sense of the word. For instance $R_{S v}$ transforms a "supervector" $v^{\infty}-v$ whose components are vectors $\mathbf{v}^{\infty}\left(\mathbf{r}_{\alpha}\right)-\mathbf{v}_{\alpha}(\alpha$ $=1, \ldots, N)$, into a "supervector'" $S$ whose components are second-order tensors $\mathbf{S}_{\alpha}(\alpha$ $=1, \ldots, N)$. This is the reason why we have not introduced a special notation for "matrix multiplication" in the above equation and in further equations to come. Therefore, the particular multiplication that is implied should be clear from the context. Finally, for our discussion of suspensions of spheres in a viscoelastic medium it is convenient to display the explicit viscosity $\left(\eta_{m}\right)$ dependence in the grand resistance matrix, as is shown in Eq. (10).

The Brownian force vector $f_{B}$ is a Gaussian white-noise vector process with a correlation matrix that reflects the hydrodynamic interactions between the particles [Tough et al. (1987)]. It is defined by

$$
\begin{gathered}
\left\langle f_{B}(t)\right\rangle=0 \\
\left\langle f_{B}(t) f_{B}\left(t^{\prime}\right)\right\rangle=2 k_{B} T \eta_{m} R_{f v}(t) \delta\left(t-t^{\prime}\right),
\end{gathered}
$$

where $R_{f v}(t) \equiv R_{f v}[r(t)]$ with $r(t) \equiv\left[r_{1}(t), \ldots, r_{N}(t)\right]^{T}$ the vector of the particle positions. In the Stokesian dynamics method this Langevin equation is considered in the so-called high-friction, or overdamped, limit. In this limit the velocity of each particle relaxes very fast to a value dictated by thermodynamic equilibrium at temperature $T$. This means that the dynamics of the particles, described by Eq. (8) only involve their positions and no longer their velocities. This contracted dynamics is again described by a Langevin equation which follows from Eq. (8) by a subtle elimination procedure [see, for instance, Sancho, San Miguel, and Dürr (1982)]. The leading order result in the limit of high friction is given by

$$
f_{H}+k_{B} T R_{f v} \nabla\left(R_{f v}\right)^{-1}+f_{B}=0 .
$$

With the help of Eq. (10) we can rewrite Eq. (12) as

$$
\frac{d r}{d t}(t)=v^{\infty}(t)+\frac{k_{B} T}{\eta_{m}} \nabla\left[R_{f v}(t)\right]^{-1}+\left[R_{f v}(t)\right]^{-1}\left(R_{f D}(t) D^{\infty}(t)+\frac{1}{\eta_{m}} f_{B}(t)\right) .
$$

As a minor technical point we should like to add that this last equation should be interpreted in the Itô sense [see, for instance, van Kampen (1981)] in order to be consistent and meaningful. In the Stokesian dynamics algorithm Eq. (13) is integrated to first order in $\Delta t$ to obtain the particle displacements. This $\Delta t$ should be larger than the Smoluchowski or Brownian time scale $t_{B} \equiv m /\left(6 \pi \eta_{m} a\right)$, but should be smaller than the typical time scale in which the structure of the suspension changes significantly, i.e., $t_{D} \equiv a^{2} / D$, where $D$ denotes the self-diffusion coefficient of a particle. This leads to the condition

$$
t_{B}<\Delta t<t_{D}
$$

A table of typical values for $t_{B}$ and $t_{D}$ can be found in the work of Nägele (1996). When the suspension is exposed to an oscillating shear strain in addition, this $\Delta t$ should be significantly smaller than a period of the oscillation: 


$$
\Delta t \ll T_{\text {osc }} .
$$

To calculate the particle contribution to the macroscopic stress tensor $\mathbf{T}_{p}$, this $\mathbf{T}_{p}$ is split into a hydrodynamic part and a Brownian part. The hydrodynamic part is given by

$$
\mathbf{T}_{p, H}(t)=\sum_{\alpha} \mathbf{S}_{H, \alpha}(t)=\sum_{\alpha}\left\{R_{S v}(t)\left[v^{\infty}(t)-v_{H}(t)\right]-R_{\mathrm{SD}}(t) D^{\infty}(t)\right\}_{\alpha},
$$

where $v_{H}$ denotes the hydrodynamic velocity which is given by the right-hand side of Eq. (12) with $f_{B}=0$ and $\nabla R_{f v}^{-1}=0$. The Brownian part is of the following form [Bossis and Brady (1989); Jongschaap and Mellema (1995)]:

$$
\mathbf{T}_{B}(t)=-\frac{1}{V} \sum_{\alpha}\left[\mathbf{S}_{B, \alpha}(t)+\mathbf{r}_{\alpha}(t) \mathbf{f}_{\alpha}(t)\right]=-k_{B} T \sum_{\alpha} \nabla\left[R_{S v}(t) R_{f v}^{-1}(t)\right]_{\alpha},
$$

in which $\mathbf{S}_{B, \alpha}$ represents the Brownian contribution to the stresslet associated with particle $\alpha$. In the simulations the macroscopic stress tensor is calculated at the end of each time step $\Delta t$. In what follows we will focus on the $x y$ component of $\mathbf{T}_{p}$. When the suspension is subjected to an oscillating shear strain $\gamma=\gamma_{0} \sin \omega t$, the component $\mathbf{T}_{p, x y}$ will also oscillate and can be represented by [see, for instance, Macosko (1994)]

$$
\mathbf{T}_{p, x y}(t)=\gamma_{0} G_{p}^{\prime}(\omega) \sin \omega t+\gamma_{0} G_{p}^{\prime \prime}(\omega) \cos \omega t,
$$

where $G_{p}^{\prime}$ represents the particle contribution to the elastic modulus and $G_{p}^{\prime \prime}$ the particle contribution to the viscous modulus. Both quantities can be calculated using [Heyes (1994)]

$$
\begin{aligned}
& G_{p}^{\prime}(\omega)=\frac{\omega}{\gamma_{0} \pi} \int_{0}^{2 \pi / \omega} d t \mathbf{T}_{p, x y}(t) \sin \omega t, \\
& G_{p}^{\prime \prime}(\omega)=\frac{\omega}{\gamma_{0} \pi} \int_{0}^{2 \pi / \omega} d t \mathbf{T}_{p, x y}(t) \cos \omega t .
\end{aligned}
$$

From this $G_{p}^{\prime}(\omega)$ and $G_{p}^{\prime \prime}(\omega)$ the real and imaginary components of the particle contribution to the complex viscosity, i.e., $\eta_{p}(\omega) \equiv \eta_{p}^{\prime}(\omega)-i \eta_{p}^{\prime \prime}(\omega)$ can be determined by

$$
\begin{aligned}
& \eta_{p}^{\prime}(\omega)=\frac{1}{\omega} G_{p}^{\prime \prime}(\omega), \\
& \eta_{p}^{\prime \prime}(\omega)=\frac{1}{\omega} G_{p}^{\prime}(\omega) .
\end{aligned}
$$

Finally, by adding the medium viscosity $\eta_{m}$ to this $\eta_{p}$ we obtain the total shear viscosity of the suspension.

\section{Stokesian dynamics of spheres in a Maxwell medium}

As was mentioned in Sec. I, we will restrict the discussion to the case where the suspension as a whole displays linear viscoelastic behavior. This is equivalent to a situation where the radius of the spheres $a$ and their overall typical velocity $U$ are such that both the Reynolds and Weissenberg numbers are very small, i.e.,

$$
\operatorname{Re} \equiv \rho a U / \eta_{m} \ll 1, \quad W e \equiv \tau U / a \ll 1,
$$


where $\rho$ is the mass density of the fluid. The first of these conditions implies that the balance of momentum in the Maxwell medium reduces to that of creeping flow,

$$
\nabla \cdot \sigma(\mathbf{r}, t)=0,
$$

while the second condition implies that the elastic response of the medium is assumed to be small. For a Newtonian fluid Eq. (21) leads to

$$
\nabla p(\mathbf{r}, t)=2 \eta_{m} \nabla \cdot \mathbf{D}(\mathbf{r}, t) .
$$

In order to determine how the viscoelasticity of the suspending medium affects the flow around the particles we study the temporal Fourier transform of Eq. (21) in combination with the Fourier transform of Eq. (1) and that of Eq. (2). For spheres in a viscous fluid this leads to

$$
\nabla \tilde{p}(\mathbf{r}, \omega)=2 \eta_{m} \nabla \cdot \tilde{\mathbf{D}}(\mathbf{r}, \omega),
$$

while for spheres in a Maxwell medium we find

$$
\nabla \tilde{p}(\mathbf{r}, \omega)=2 \eta_{m}^{*} \nabla \cdot \tilde{\mathbf{D}}(\mathbf{r}, \omega), \quad \text { with } \quad \eta_{m}^{*}=\frac{\eta_{m}}{1+i \omega \tau}
$$

where in accordance with Eq. (20), we have made use of the fact that $d / d t$ can be approximated by $\partial / \partial t$. Equations (23) and (24) have the same structure. This implies that the boundary value problems for viscous and linear viscoelastic fluids have similar solutions. This is known as the "correspondence principle" [see, for instance, Christensen (1971)]. By using this principle one can derive the analog of Eq. (10) for particles suspended in a linear viscoelastic medium in the following way. For instance, if we substitute the Fourier transform of Eq. (2), i.e., $\tilde{\sigma}^{\prime}(\mathbf{r}, \omega)=2 \eta_{m}^{*} \nabla \cdot \tilde{\mathbf{D}}(\mathbf{r}, \omega)$, into the Fourier transform of Eq. (9), the result is $\tilde{\mathbf{f}}_{H, \alpha}(\omega)=2 \eta_{m}^{*} \int_{A_{\alpha}} d^{2} r \tilde{\mathbf{D}}(\mathbf{r}, \omega)$. From the correspondence principle it follows that the right-hand side of this result is equal to $\eta_{m}^{*}$ times the Fourier transform of the hydrodynamic force on particle $\alpha$ in a viscous fluid with a unit viscosity. A similar result can be derived for $\tilde{\mathbf{S}}_{\alpha}(\mathbf{r}, \omega)$. With the help of Eq. (10) this then leads to

$$
\left(\begin{array}{c}
\tilde{f}_{H} \\
\tilde{S}
\end{array}\right)=\eta_{m}^{*} \mathcal{F}\left[\left(\begin{array}{ll}
R_{f v} & R_{f D} \\
R_{S v} & R_{\mathrm{SD}}
\end{array}\right)\left(\begin{array}{c}
v^{\infty}-v \\
D^{\infty}
\end{array}\right)\right] .
$$

The validity of this relationship, in addition, rests upon the fact that on the time scale of interest, namely, that for $t_{B}=m /\left(6 \pi \eta_{m} a\right) \ll t \leqslant \tau$, one can neglect particle momentum relaxation and particle inertia (see the Appendix). This implies that in this regime the dynamics of the particles is, similar to the viscous case, again described by an overdamped Langevin equation and that the hydrodynamic interaction between the particles only depends on their relative positions. Multiplication of Eq. (25) by $(1+i \omega \tau)$ followed by an inverse Fourier transformation back to real time yields

$$
\left(\begin{array}{c}
\tau \frac{d}{d t}+1
\end{array}\right)\left(\begin{array}{c}
f_{H} \\
S
\end{array}\right)=\eta_{m}\left(\begin{array}{ll}
R_{f v} & R_{f D} \\
R_{S v} & R_{\mathrm{SD}}
\end{array}\right)\left(\begin{array}{c}
v^{\infty}-v \\
D^{\infty}
\end{array}\right) .
$$

In the simulations this equation is numerically solved by time stepping. Equation (26) plays a central role in the remainder. We will now show how in the high-friction limit viscoelasticity affects the calculation of the particle displacements. To that end let us first consider how the Brownian force $f_{B}$ is to be defined in a Maxwell medium. For a dilute 
suspension of particles in a Maxwell medium, where hydrodynamic interactions between the particles are absent, $f_{B}$ will be non-Gaussian and satisfies [Kubo et al. (1991); Volkov and Vinogradov (1984)]

$$
\begin{gathered}
\left\langle f_{B}(t)\right\rangle=0, \\
\left\langle f_{B}(t) f_{B}\left(t^{\prime}\right)\right\rangle=\frac{k T}{\tau} \zeta e^{-\left|t-t^{\prime}\right| / \tau} I
\end{gathered}
$$

in order for the dispersion under quiescent conditions to ultimately attain a proper thermodynamic equilibrium state. Equation (27) is a consequence of the fluctuationdissipation theorem [Kubo et al. (1991)]. In Eq. (27) we have introduced $\zeta$ to represent the friction of an individual particle. Thus the Brownian force is colored in this case. In fact $f_{B}$ is a so-called Ornstein-Uhlenbeck stochastic process [see, for instance, van Kampen (1981)]. Therefore it follows that $f_{B}$ obeys the following stochastic differential equation:

$$
\tau \frac{d f_{B}(t)}{d t}+f_{B}(t)=\sqrt{k_{B} T \zeta} \xi(t),
$$

where $\xi(t)$ is a Gaussian white noise process with properties of

$$
\begin{gathered}
\langle\xi(t)\rangle=0, \\
\left\langle\xi(t) \xi\left(t^{\prime}\right)\right\rangle=2 \delta\left(t-t^{\prime}\right) I .
\end{gathered}
$$

When hydrodynamic interactions cannot be neglected between the particles, a generalization of Eq. (28) reads as follows:

$$
\tau \frac{d f_{B}(t)}{d t}+f_{B}(t)=\sqrt{k_{B} T \eta_{m}} g(t) \xi(t),
$$

with the $3 N \times 3 N$ matrix $g(t)$ such that $R_{f v}(t) \equiv g(t) g(t)^{T}$.

The full dynamics of the suspension is still described by Eq. (8) but now with $f_{H}$ and $f_{B}$ satisfying Eqs. (26) and (30), respectively. By combining the time derivative of Eq. (8) with Eqs. (26) and (30) one can derive the following Langevin equation:

$$
m \tau \frac{d a}{d t}=-m a+\eta_{m}\left[R_{f v}\left(v^{\infty}-v\right)+R_{f D} D^{\infty}\right]+\sqrt{k_{B} T \eta_{m}} g \xi,
$$

with $a \equiv d v / d t$, the vector of the particle accelerations, and off course with $v$ $\equiv d r / d t$. Thus, the full dynamics can be described as taking place in an extended $9 N$-dimensional phase space $\Gamma=\left[(r, v, a)^{T}\right]$. This idea was introduced by Volkov and Leonov (1996) in their treatment of a single Brownian particle in a Maxwell medium. The overdamped Langevin equation that describes the dynamics of the system on the time scale of interest $t_{B} \ll t \leqslant \tau$ mentioned earlier can be derived from Eq. (31) by generalization of the adiabatic elimination procedure which leads from Eq. (8) to Eq. (12) (see the Appendix). On this time scale it is possible to eliminate both the acceleration $a$ and the velocity $v$ in the limit of large friction. The final result turns out to be

$$
\frac{d r}{d t}(t)=v^{\infty}(t)+\frac{k_{B} T}{\eta_{m}} \nabla\left[R_{f v}(t)\right]^{-1}+\left[R_{f v}(t)\right]^{-1}\left(R_{f D}(t) D^{\infty}(t)+\sqrt{\frac{k_{B} T}{\eta_{m}}} g(t) \xi(t)\right),
$$


which is a Langevin equation in configuration space that is identical to Eq. (13). This result implies that when particle momentum relaxation and particle inertia can be "neglected," the trajectories of the particles in a Maxwell fluid with one relaxation time are statistically indistinguishable from the corresponding trajectories in a viscous fluid. The particle displacements, as we mentioned before, can be obtained from Eq. (32) by an integration over $\Delta t$.

Next we consider the calculation of the particle contribution to the macroscopic stress tensor. From Eq. (26) it follows that the hydrodynamic contribution to the vector of stresslets obeys the differential equation,

$$
\tau \frac{d S_{H}(t)}{d t}+S_{H}(t)=\eta_{m}\left\{R_{S v}(t)\left[v^{\infty}(t)-v_{H}(t)\right]-R_{\mathrm{SD}}(t) D^{\infty}(t)\right\},
$$

where we have again used the hydrodynamic contribution to the particle velocity $v_{H}$, which is defined in the text following Eq. (15). The solution of Eq. (33) is given by

$$
\begin{aligned}
& \mathbf{S}_{H, \alpha}\left(t_{N}\right)=e^{-\Delta t / \tau} \mathbf{S}_{H, \alpha}\left(t_{N-1}\right)+\frac{1}{\tau} \int_{t_{N-1}}^{t_{N}} d t^{\prime} e^{-\left(t_{N}-t^{\prime}\right) / \tau} \\
& \times \eta_{m}\left\{R_{S v}\left(t^{\prime}\right)\left[v^{\infty}\left(t^{\prime}\right)-v_{H}\left(t^{\prime}\right)\right]-R_{\mathrm{SD}}\left(t^{\prime}\right) D^{\infty}\left(t^{\prime}\right)\right\}_{\alpha} .
\end{aligned}
$$

The first term on the right-hand side of Eq. (34) contains the long time memory contributions to the hydrodynamic stresslet, while the integral accounts for the changes of the term $\left\{R_{S v}(t)\left[v^{\infty}(t)-v_{H}(t)\right]-R_{S D}(t) D^{\infty}(t)\right\}_{\alpha}$ in the short time interval between $t_{N-1}$ and $t_{N}$. In the simulations Eq. (34) is evaluated numerically to obtain the stresslets of each of the individual particles at discrete times $t_{k}=k \Delta t(k=1,2, \ldots$,$) . The hydrody-$ namic part of the particle contribution to the bulk stress tensor $\mathbf{T}_{p, H}\left(t_{k}\right)$ at time $t_{k}$ is then determined from

$$
\mathbf{T}_{p, H}\left(t_{k}\right)=\sum_{\alpha} \mathbf{S}_{H, \alpha}\left(t_{k}\right) .
$$

Since the Brownian motion of the particles in the Maxwell medium is identical with that in the viscous medium, it follows that the Brownian contribution to the bulk stress tensor $\mathbf{T}_{B}\left(t_{k}\right)$ is again given by Eq. (16) evaluated at $t=t_{k}$. For evaluation of the particle contribution to the viscosity we use Eqs. (18) and (19). The fluid contribution to the complex viscosity is calculated directly from the temporal Fourier transform of Eqs. (2) and (5), i.e.,

$$
\begin{aligned}
& \eta_{m}^{\prime}(\omega)=1 \frac{1}{1+\omega^{2} \tau^{2}} \eta_{m}(\omega=0), \\
& \eta_{m}^{\prime \prime}(\omega)=\frac{\omega \tau}{1+\omega^{2} \tau^{2}} \eta_{m}(\omega=0) .
\end{aligned}
$$

We end this discussion by noting that in the viscoelastic case the time step $\Delta t$ should also fulfill the condition $\Delta t<\tau$ in addition to the conditions stated in Eq. (14).

\section{SIMULATION DETAILS}

The program that we used for our simulations of suspensions in three dimensions is basically the program written and described by Phung et al. (1993 and 1996). For our purposes we needed to modify the program so that suspensions subjected to an oscillating 
shear strain could be simulated. In addition we had to implement Eqs. (18) and (19) to calculate the dynamic moduli and viscosities.

The hydrodynamic interaction among different particles is calculated in the same manner as that described in the work of Phung et al. (1993 and 1996), and references therein. In our simulations, in which we have used no repulsive colloidal forces, numerical errors can cause the particles to overlap. A detailed description how the algorithm corrects this erroneous overlap can be found in the work of Bossis and Brady (1984).

The Stokesian dynamics algorithm that is used here involves $\mathcal{O}\left(N^{3}\right)$ operations, where $N$ is the number of spheres [Phung et al. (1996)]. As a result, only systems with a small number of particles can be dealt with. In our simulations we have considered systems consisting of 27 spheres. Details concerning the particle number dependence can be found in the work of Phung et al. (1993 and 1996). In our simulations we have used the diffusion time $t_{D}=a^{2} / D$ to make the times and frequencies in this article dimensionless. The time step used in the simulations was chosen so that one period of the imposed oscillating shear consisted of at least 500 time steps. In the simulations this boils down to $0.00005 \leqslant \Delta t \leqslant 0.0005$, where the smallest values for $\Delta t$ were used in the simulations of the highest frequencies. The number of time steps in a single simulation varied between 80000 and 800000 . Both the low-frequency simulations and the high-frequency simulations required a large number of time steps. The reason for this is as follows. For the low-frequency simulations we have $\Delta t<t_{D}<T_{\text {osc }}$. In this case we need a large number of time steps to complete one period of the oscillation. In the high-frequency regime we have small values for $T_{\text {osc }}$ but, because of $\Delta t<T_{\text {osc }}$, we need a large number of steps to reach a time scale that is sufficiently large for equilibration and data sampling. The maximum strain amplitude was taken to be $\gamma_{0}=0.02$. Test runs have shown this value of $\gamma_{0}$ to be in the linear regime. For each frequency the response of the system was evaluated in at least seven independent runs. In each run the results obtained during the first periods of imposed strain were discarded in order to avoid startup artifacts in the results. In Fig. 1 we show the time dependence of $\Sigma_{\alpha} \mathbf{S}_{H, \alpha}(t) \cos \omega t$ for suspensions in a viscous $(\tau=0)$ and a Maxwell fluid $(\tau=0.5)$ subjected to an oscillating shear strain. In the absence of Brownian motion it is found that for a suspension with a viscous matrix $\Sigma_{\alpha} \mathbf{S}_{H, \alpha}(t) \cos \omega t$ is, to good approximation, proportional to $\cos ^{2} \omega t$. Incorporation of Brownian motion causes the amplitude of this quantity to fluctuate in time. For a suspension with a Maxwell matrix we find that $\Sigma_{\alpha} \mathbf{S}_{H, \alpha}(t)$ has undergone a phase shift due to the viscoelasticity of the medium. On the basis of the results presented in Fig. 1 it is to be expected that the values for the hydrodynamic contribution to $G_{p}(\omega)$ will be influenced by the presence of Brownian motion.

\section{RESULTS AND DISCUSSION}

Here we present the results obtained by Stokesian dynamics simulations of suspensions subjected to an oscillating shear strain. First we will discuss results concerning suspensions with a Newtonian matrix. These results complement the results obtained by Phung et al. (1993 and 1996), who have considered suspensions with a viscous matrix subjected to simple shear. Furthermore, these new results will serve as a reference in our discussion of the results obtained for spheres in a Maxwell medium, with which we will conclude Sec. IV.

\section{A. Suspensions of spheres in a viscous medium}

Figure 2 illustrates how the viscosity of a suspension subjected to an oscillating shear strain with $\omega=2 \pi$ depends on the volume fraction $\phi$ of the spheres. The angular 

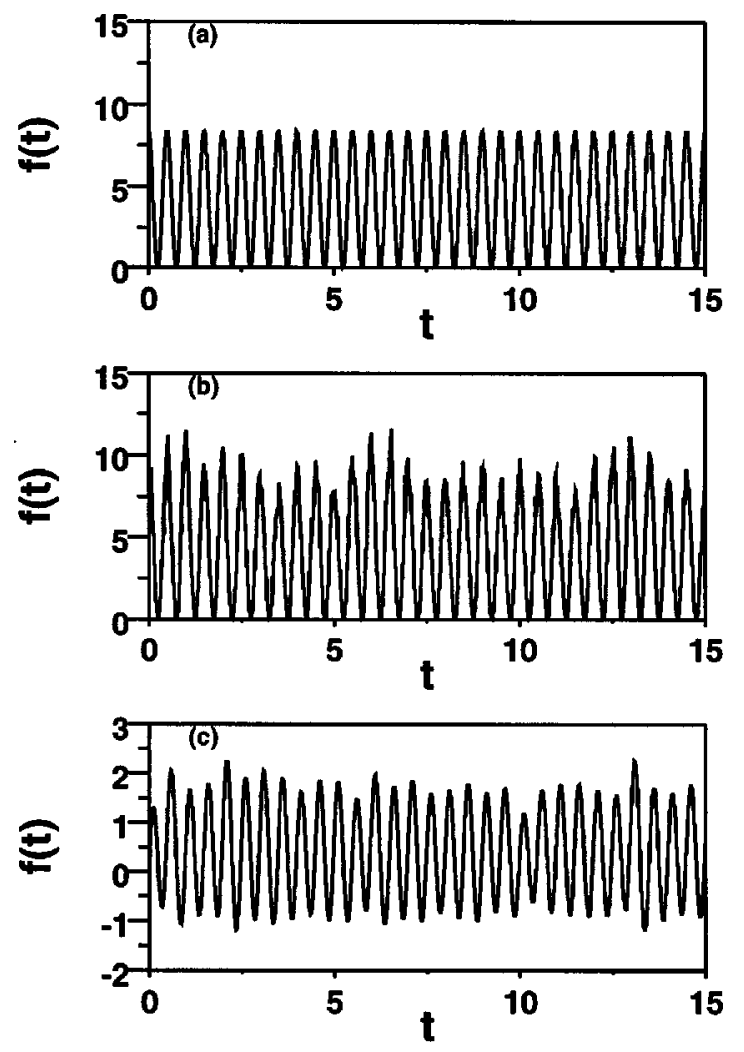

FIG. 1. Time dependence of $f(t)=\Sigma_{\alpha} \mathbf{S}_{H, \alpha}(t) \cos \omega t$ for (a) particles in a viscous medium with no Brownian motion, (b) particles in a viscous medium with Brownian motion, and (c) particles in a Maxwell medium ( $\tau$ $=0.5)$ with Brownian motion.

frequency used here is made dimensionless with $t_{D}$. The numerical values for the viscosities and the elastic and viscous moduli presented here in Sec. IV are reduced with respect to $\eta_{m}$. Also shown in Fig. 2 is the sum of the matrix and hydrodynamic contributions to the viscosity $\eta_{m}+\eta_{H}$. It can be seen that at low volume fractions the total viscosity is given by $\eta^{\prime} \approx \eta_{m}+\eta_{H}$. At these volume fractions we find that the relation of Batchelor and Green (1972),

$$
\eta^{\prime}=\eta_{m}\left(1+\frac{5}{2} \phi+5.2 \phi^{2}\right),
$$

is obeyed well. Equation (37) is valid for systems without Brownian motion [Batchelor and Green (1972)]. At higher volume fractions the contribution to the viscosity due to Brownian motion increases significantly. At these volume fractions Eq. (37) is no longer sufficient to describe $\eta^{\prime}$. Since the values for $\eta_{H}$ obtained here are also relevant for our discussion of the complex viscosity of suspensions of spheres in Maxwell media, given in Sec. IV B, it is useful to present our results for $\eta_{H}$ by the following polynomial fit:

$$
\eta_{H}=\eta_{m}\left(\frac{5}{2} \phi+5.2 \phi^{2}+3.01 \phi^{3}+47.71 \phi^{4}\right)
$$

for volume fractions in the range of $0 \leqslant \phi \leqslant 0.45$. Equation (38) reproduces our Stokesian dynamics results for $\eta_{H}$ within 1\%. In Fig. 3 we compare the predictions of Eq. (38) to the experimental results for $\eta^{\infty}=\eta_{H}+1$ obtained by van der Werff et al. (1989) and 


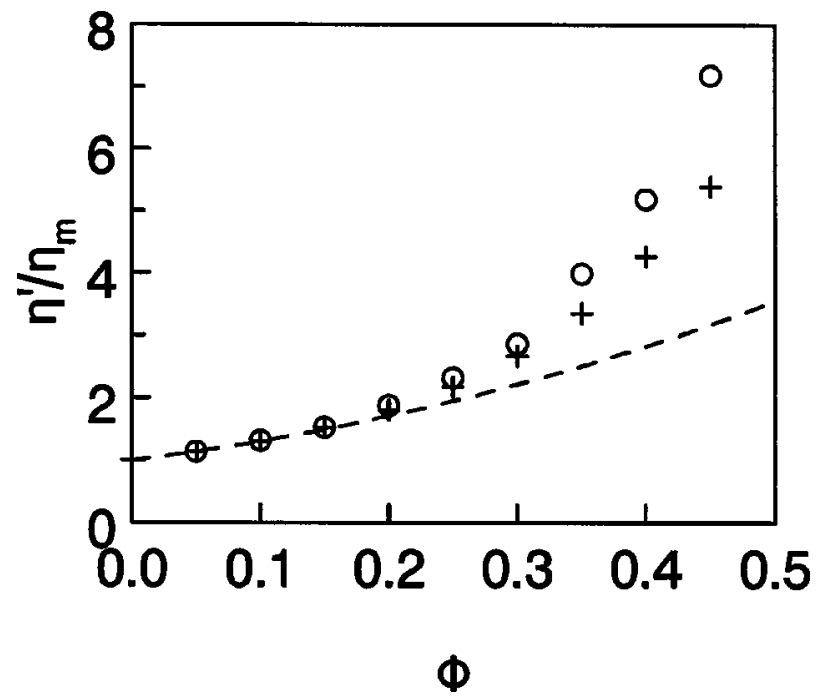

FIG. 2. Sphere volume fraction dependence of the viscosity determined by Stokesian dynamics at $\omega=6 \pi$; + : $1+\eta_{H} / \eta_{m} ; \bigcirc: 1+\eta_{H} / \eta_{m}+\eta_{B} / \eta_{m} ;$ dashed line: Batchelor equation.

by Shitaka and Pearson (1994). It can be seen that good agreement is found between the simulations and experiments. The $\eta_{H}$ value calculated using Eq. (38) for $\phi=0.45$ is within $1 \%$, equal to the $\eta_{H}$ value obtained by interpolating the Peclet number dependence of the steady shear results obtained by Phung at al. (1993 and 1996). Finally, it can be seen in Fig. 3 that the Stokesian dynamics results, as represented by Eq. (38), are systematically larger than the theoretical approximation used by Verberg et al. (1997).

Next we come to a discussion of the viscoelastic properties of rigid spheres in a viscous medium. A detailed description of the mechanism that gives rise to the viscoelas-

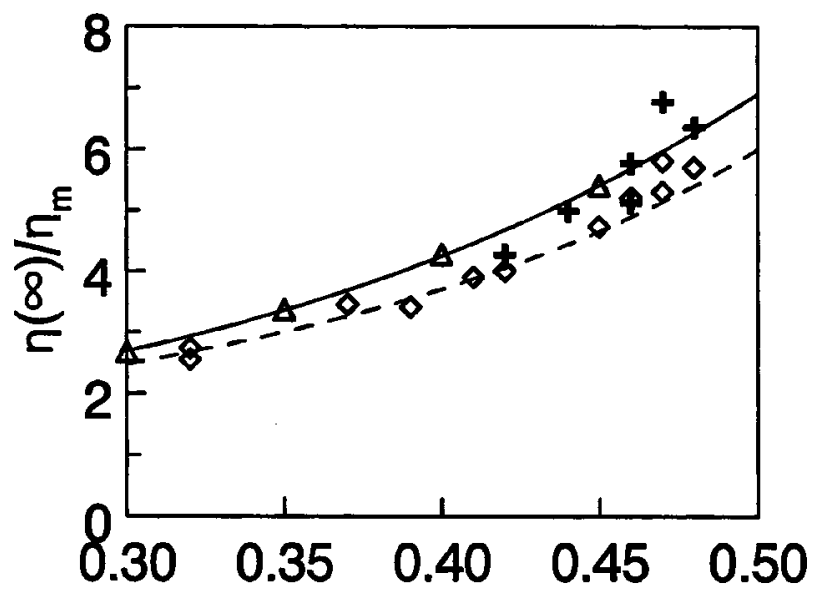

$\Phi$

FIG. 3. Stokesian dynamics value of $1+\eta_{H} / \eta_{m}(\triangle$ and solid line) compared with the experimental results of van der Werff et al. (1989) (+) and Shikata and Pearson (1994) $(\diamond)$, and the theoretical prediction of Verberg et al. (1997) (dashed line). 


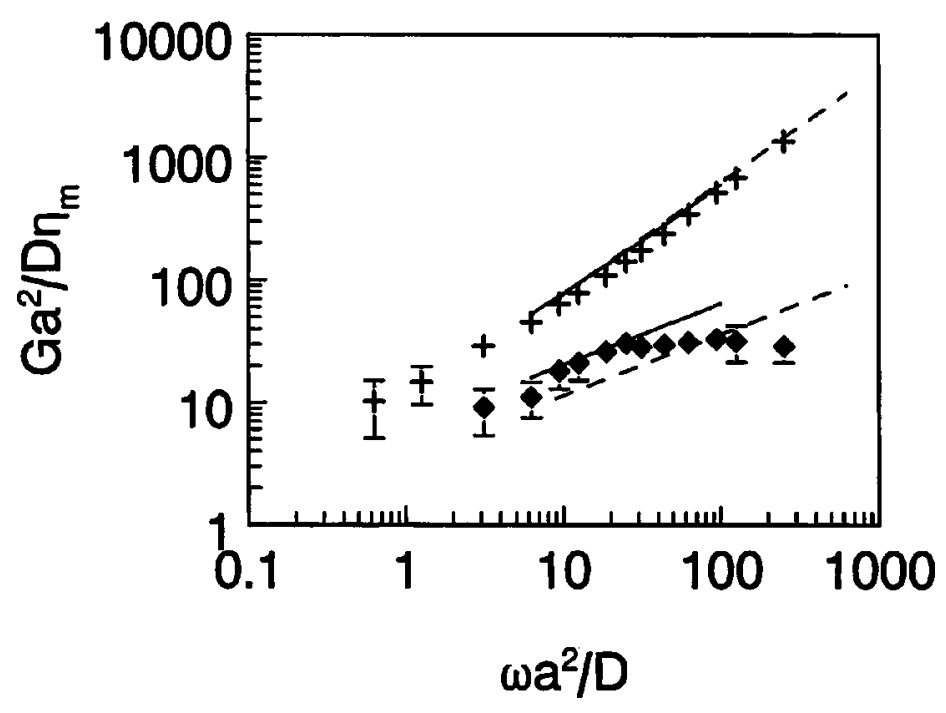

FIG. 4. Frequency dependence of the viscous modulus $G^{\prime \prime}(+)$ and the elastic modulus $G^{\prime}(\diamond)$ obtained by SD for a suspension of spheres in a viscous medium $(\phi=0.45)$ compared with the $\omega^{-1 / 2}$ fits to the experimental results of van der Werff et al. (1989) (solid line: $s p 23, \phi=0.46$; dashed line: $s s f 1, \phi=0.44$ ).

ticity of these suspensions can be found in the paper of van der Werff et al. (1989). It should be noted that the mechanism that gives rise to the viscoelasticity of these suspensions is already present in the theory of Batchelor (1977), as was pointed out by Mellema et al. (1987). In Fig. 4 we show the elastic and viscous moduli calculated using Stokesian dynamics for a dispersion with $\phi=0.45$. It can clearly be seen that the viscous modulus $G^{\prime}$ attains a plateau value for $\omega>8 \pi$. A similar plateau for $G^{\prime}$ was also reported by Shikata and Pearson (1994). In the paper by van der Werff et al. (1989), such a plateau is not reported, although their experiments were done in the frequency range where our plateau is observed. The experiments of van der Werff et al. have shown that the viscous and the elastic contributions to the complex viscosity have an $\omega^{-1 / 2}$ dependence. In Fig. 5 we show the extent to which our data show this behavior. For $\eta^{\prime \prime}$ we find good agreement with the experimental results of van der Werff et al., while for $\eta^{\prime}$ our results are systematically lower than the experimental values. For small $\omega$ we find that $\eta^{\prime}$ is constant. The experimental data presented by van der Werff et al. suggest a similar deviation from the $\omega^{-1 / 2}$ dependence. This deviation might be related to the possible existence of a high-frequency plateau for $G^{\prime}$.

We have performed some Stokesian dynamics simulations in order to study the appearance of the plateau of $G^{\prime}$ at volume fractions $\phi=0.4$ and 0.48 . These calculations have revealed a frequency dependence of $G^{\prime}$ that is similar to the results presented in Fig. 4. A comparison of the high-frequency modulus $G_{\infty}^{\prime}$ obtained by Stokesian dynamics on the one hand and experimental results [Shikata and Pearson (1994)] and a theoretical prediction [Lionberger and Russel (1994)] on the other is given in Fig. 6.

One may wonder to what extent we are justified to perform Stokesian dynamics simulations in the frequency range considered here. The Stokesian dynamics technique is valid as long as the inertial effects can be neglected [see Eq. (9)]. Schnur et al. (1997) have shown that inertial effects can be neglected for frequencies for which the inertial decay length is much larger than the sphere radius, i.e., $1 / \Gamma=\left(2 G^{*} / \rho \omega^{2}\right)^{1 / 2} \gg a$. When we plug in our values of $G^{*}$, in combination with the data for $a$, the temperature 


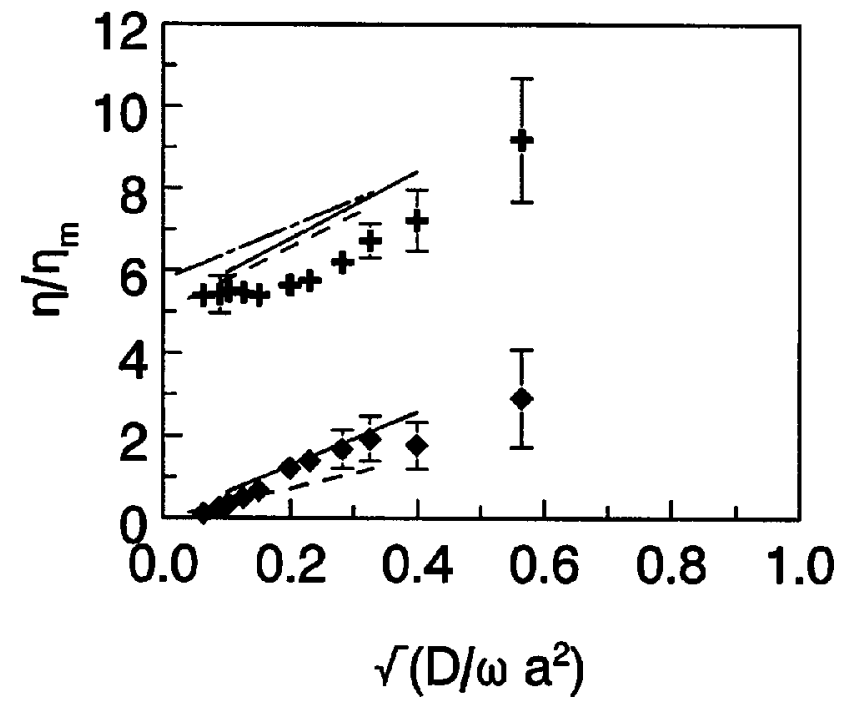

FIG. 5. $\omega^{-1 / 2}$-dependence of the complex viscosity $\left(+: \eta^{\prime} / \eta_{m} ;: \eta^{\prime \prime} / \eta_{m}\right)$ obtained by SD for a suspension of spheres in a viscous medium ( $\phi=0.45)$ compared with the linear fits to the experimental results of van der Werff et al. (1989) (solid line: $s p 23, \phi=0.46$; dashed-dotted line: $s j 18 \phi=0.46$; dashed line: $s s f 1, \phi$ $=0.44)$.

and the viscosity of the medium given by van der Werff et al. (1989), we find that this condition holds in all our simulations. Thus, inertial effects are expected to appear at considerably higher frequencies.

We conclude Sec. IV A by presenting in Fig. 7 the frequency dependence of the complex viscosity and its hydrodynamic contribution. We find that the hydrodynamic

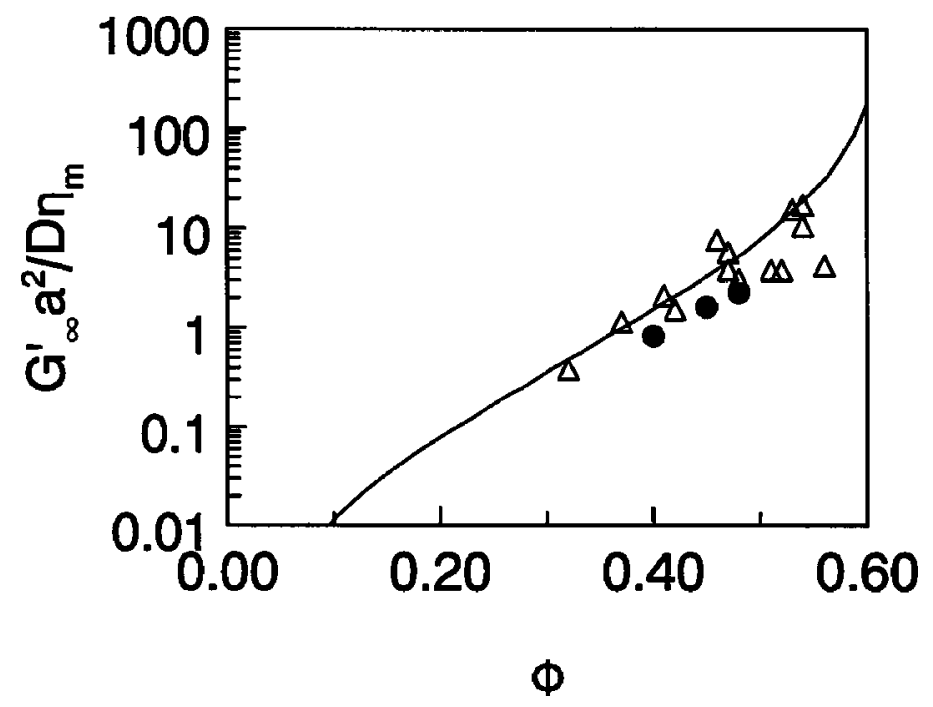

FIG. 6. High-frequency modulus as a function of the volume fraction; $\triangle$ : experimental data of Shikata and Pearson (1994) (reduced using the hydrodynamic radius $a_{H}$ ); solid line: theoretical prediction of Lionberger and Russel (1994); •: results obtained by Stokesian dynamics. 

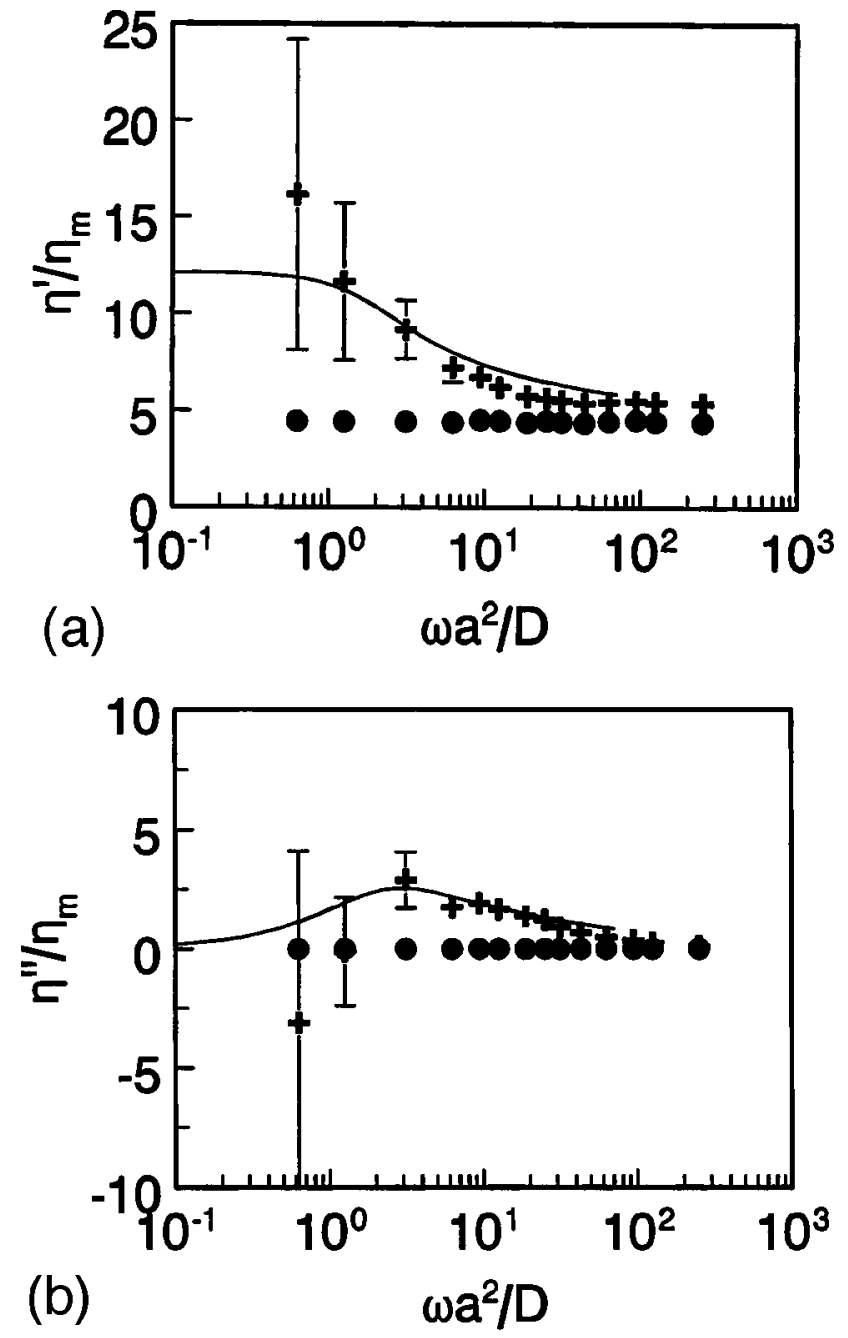

FIG. 7. Frequency dependence of the complex viscosity obtained by Stokesian dynamics for a suspension of spheres in a viscous medium $(\phi=0.45) ; \bullet: \eta_{H} ;+: \eta=\eta_{m}+\eta_{H}+\eta_{B}$; solid line: experimental results of van der Werff et al. (1989) (ssf1, $\phi=0.44$ ). (a) $\eta^{\prime} / \eta_{m}$; (b) $\eta^{\prime \prime} / \eta_{m}$.

contribution to the complex viscosity is constant within $2 \%$ for all frequencies. The frequency dependence of $\eta^{*}(\omega)$ compares well with the fit obtained by van der Werff et al. (1989).

\section{B. Suspensions of spheres in a Maxwell medium}

In Fig. 7 we show the frequency dependence of the complex viscosity for a suspension of spheres in a Maxwell medium characterized by a single relaxation time $\tau=0.5$. For comparison we also show in Fig. 8 the frequency dependence of the viscosity of the pure Maxwell medium itself. It is seen that the addition of spheres to the viscoelastic medium causes a significant increase of $\eta^{\prime}(\omega)$ and $\eta^{\prime \prime}(\omega)$, in comparison to the pure medium values. This increase of the complex viscosity is also observed experimentally [Chan and Powell (1984); Aral and Kaylon (1997)]. As for suspensions with a viscous matrix, we 

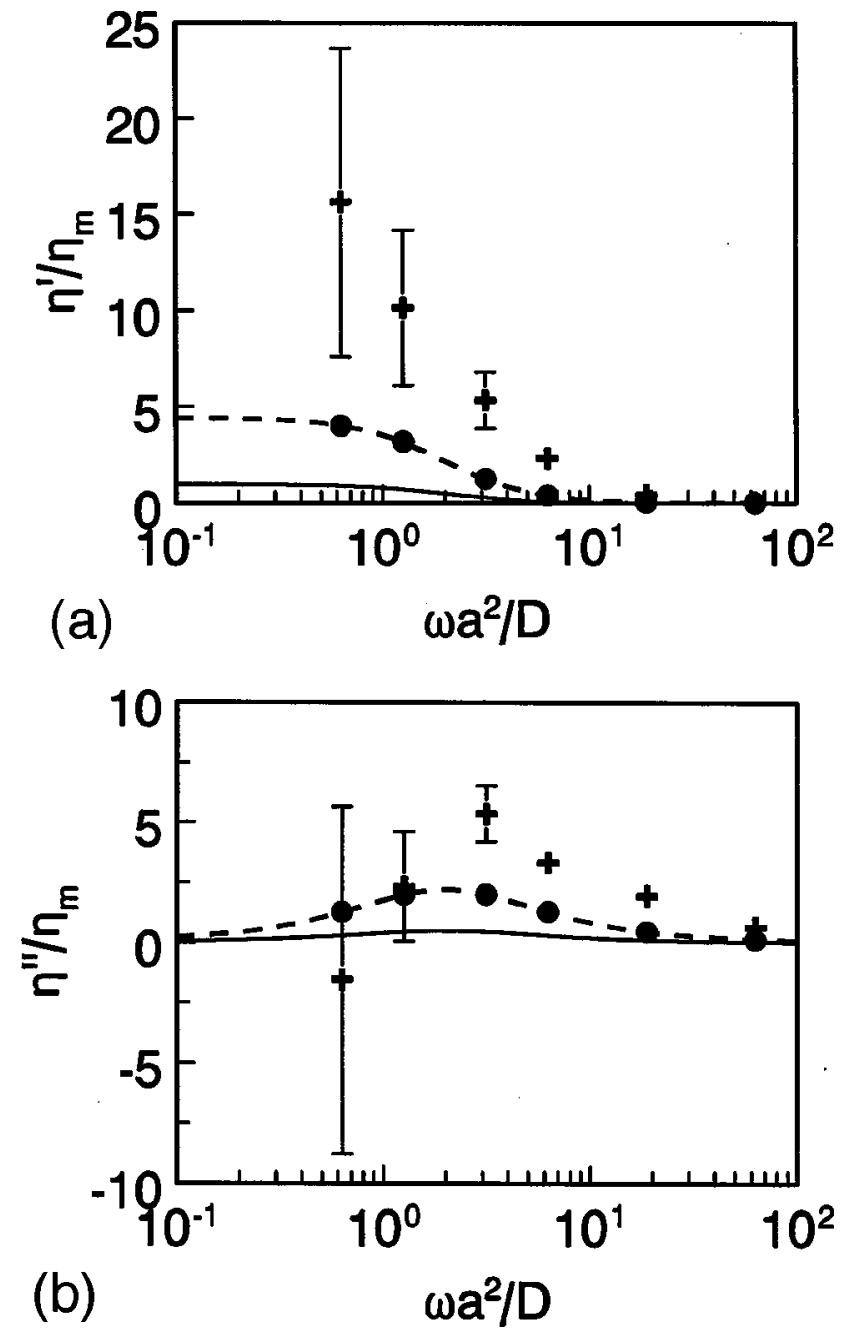

FIG. 8. Frequency dependence of the complex viscosity for a suspension of spheres in a Maxwell medium $(\phi=0.45, \tau=0.5)$; solid line: $\eta_{m}(\omega)$; + : SD results for $\eta_{m}(\omega)+\eta_{H}(\omega)$; dashed line: Eq. (41); $\eta_{m}(\omega)+\eta_{H}(\omega)+\eta_{B}(\omega)$. (a) $\eta^{\prime} / \eta_{m}$; (b) $\eta^{\prime \prime} / \eta_{m}$.

find that for concentrated suspensions with a Maxwell matrix the contribution of Brownian motion is significant at low frequencies and that this contribution decreases with increasing frequency. In contrast to our results for spheres in a viscous medium we find that the hydrodynamic contribution to the viscosity is no longer independent of the frequency. The frequency dependencies of the hydrodynamic contributions $\eta_{H}^{\prime}(\omega)$ and $\eta_{H}^{\prime \prime}(\omega)$ seem to be similar to the frequency dependencies found for the pure Maxwell fluid. In fact it is possible to describe the hydrodynamic contribution to the complex viscosity quantitatively using the results obtained for spheres in a viscous medium. To show this we take the Fourier transform of Eq. (33). The result is

$$
\mathbf{S}_{\alpha}(\omega)=\mathbf{S}_{\alpha}^{0}(\omega) \frac{1}{1+i \omega \tau}
$$


where $\mathbf{S}_{\alpha}^{0}(\omega)$ is a shorthand notation for

$$
\mathbf{S}_{\alpha}^{0}(\omega)=\int d t e^{i \omega t} \eta_{m}\left\{R_{S v}(t)\left[v^{\infty}(t)-v_{H}(t)\right]-R_{\mathrm{SD}}(t) D^{\infty}(t)\right\}_{\alpha} .
$$

From Eq. (32) we concluded that the dynamics of the particles are not affected by the viscoelasticity of the medium. Therefore, when the integrand in Eq. (40) is averaged over all particles, we expect its value to be equal to the value obtained for a system of spheres in a viscous medium (at the same volume fraction $\phi$ ). This implies that $\Sigma_{\alpha} \mathbf{S}_{\alpha}^{0}(\omega)$ of a suspension with a Maxwell matrix should be equal to the corresponding $\Sigma_{\alpha} \mathbf{S}_{\alpha}^{0}(\omega)$ of a suspension with a viscous matrix. In Fig. 6 we have seen that the hydrodynamic contribution to the viscosity for suspensions with a viscous matrix is independent of the frequency. When we apply this result to Eqs. (39) and (40), we find for the hydrodynamic contribution to the complex viscosity of a suspension with a Maxwell matrix,

$$
\begin{aligned}
& \eta_{H}^{\prime}(\omega)=\frac{1}{1+\omega^{2} \tau^{2}} \eta_{H}(\tau=0), \\
& \eta_{H}^{\prime \prime}(\omega)=\frac{\omega \tau}{1+\omega^{2} \tau^{2}} \eta_{H}(\tau=0),
\end{aligned}
$$

where the value of $\eta_{H}(\tau=0)$ can be calculated using Eq. (38). The low volume fraction limit of Eq. (41) [up to order $\mathcal{O}(\phi)$ ] was obtained earlier by Volkov and Vinogradov (1984) using a different argument. In Fig. 8 it can be seen that Eq. (41) gives a very good description of the Stokesian dynamics results for the hydrodynamic contribution to the complex viscosity. We have obtained similar agreement between theory and simulation results for $\eta_{H}$ for suspensions of spheres in Maxwell media with more relaxation times. Apparently the Brownian contributions to $\Sigma_{\alpha} \mathbf{S}_{\alpha}(t)$, observed in Fig. 1, cancel each other in the process of averaging over time. Equation (41) can be generalized to include more relaxation times:

$$
\begin{aligned}
& \eta_{H}^{\prime}(\omega)=\frac{\eta_{H}(\tau=0)}{\eta_{0, m}} \sum_{k} \frac{\eta_{k}}{1+\omega^{2} \tau_{k}^{2}}, \\
& \eta_{H}^{\prime \prime}(\omega)=\frac{\eta_{H}(\tau=0)}{\eta_{0, m}} \sum_{k} \frac{\eta_{k}}{1+\omega^{2} \tau_{k}^{2}},
\end{aligned}
$$

with $\Sigma_{k} \eta_{k}=\eta_{0, \mathrm{~m}}$.

In the literature two experimental studies have been reported in which reasonably well defined suspensions of glass spheres in a viscoelastic matrix subjected to an oscillating shear strain have been studied [Chan and Powell (1984); Aral and Kaylon (1997)]. The conditions of these experiments are summarized in Table I. By making the experimental frequency $\tilde{\omega}$ comparable to our frequency $\omega=\tilde{\omega}\left(a^{2} / D\right)=\tilde{\omega}\left(\eta_{0, m} a^{3} / k_{B} T\right)$ we find that both experiments were done in the frequency range of $10^{7} \leqslant \omega<3 \times 10^{10}$. Since a single time step in the Stokesian dynamics simulation should be considerably smaller than one period of the imposed oscillation [see Eq. (14b)], simulation of these systems would require a time step of the order of $\mathcal{O}\left(10^{-9}\right)$, or even less. This is a factor of $10^{4}$ smaller than the smallest time step used in the present study. A single run would be a factor of $10^{4}$ larger. Therefore it is at present not feasable to study these suspensions at these frequencies directly in a Stokesian dynamics simulation. For the frequency range studied in the aforementioned papers it is expected that Brownian motion is negligible 
TABLE I. Systems studied by Chan and Powell (1984) and by Aral and Kaylon (1997); the value for $\eta_{0, m}$ given for the sample reported by Aral and Kaylon was obtained by fitting a Maxwell model with three relaxation times to their data (see also Table II); the frequency shift factor of Aral and Kaylon is taken to be unity.

\begin{tabular}{|c|c|c|}
\hline Chan and Powell & $\begin{array}{c}\text { Fluid } \\
\text { Temperature } \\
\text { Sphere diameter } \\
\eta_{0, f} \\
\text { Frequencies }\end{array}$ & $\begin{array}{c}\text { NBS test sample } 1 \\
298 \mathrm{~K} \\
25 \mu \mathrm{m} \leqslant a \leqslant 38 \mu \mathrm{m} \\
67.7 \mathrm{~Pa} \mathrm{~s} \\
0.02 \pi \mathrm{rad} \mathrm{s}^{-1} \leqslant \tilde{\omega} \leqslant 20 \pi \mathrm{rad} \mathrm{s}^{-1}\end{array}$ \\
\hline Aral and Kaylon & $\begin{array}{c}\text { Fluid } \\
\text { Temperature } \\
\text { Sphere diameter } a \\
\eta_{0, f} \\
\text { Frequencies }\end{array}$ & $\begin{array}{c}\text { Polydimethylsiloxane } \\
273 \mathrm{~K} \\
6 \mu \mathrm{m} \\
96.2 \mathrm{~Pa} \mathrm{~s} \\
1.15 \mathrm{rad} \mathrm{s}^{-1} \leqslant \tilde{\omega} \leqslant 917 \mathrm{rad} \mathrm{s}^{-1}\end{array}$ \\
\hline
\end{tabular}

[Aral and Kaylon (1997)]. In this case it is possible to analyze these experiments with the help of Eqs. (38) and (42). In Fig. 9 we plot the volume fraction dependence of $\eta^{\prime}$, as observed by Chan and Powell (1984) and by Aral and Kaylon (1997) at the lowest frequencies they have considered. In our analysis of the data of Chan and Powell we have used an average sphere diameter of $31.5 \mu \mathrm{m}$. The results of Chan and Powell are in fairly good agreement with Eq. (38). This is also the case for the data of Aral and Kaylon for $\phi \leqslant 0.2$. However, for $\phi \geqslant 0.3$ we find that the results of Aral and Kaylon deviate significantly from the theoretical value.

As an example of the application of Eq. (42) to experimental data, we consider the results of Aral and Kaylon for $\phi \leqslant 0.2$. We have fitted the data for the viscous and the elastic moduli of the suspending medium (polydimethylsiloxane), taken from their paper, in terms of three Maxwell modes. The results of this fit are given in Table II. In the caption of Table II we have also given the Brownian time scale $t_{B}$ as an illustration of the separation between the viscoelastic relaxation time $\tau$ and $t_{B}$. The polydimethylsiloxane

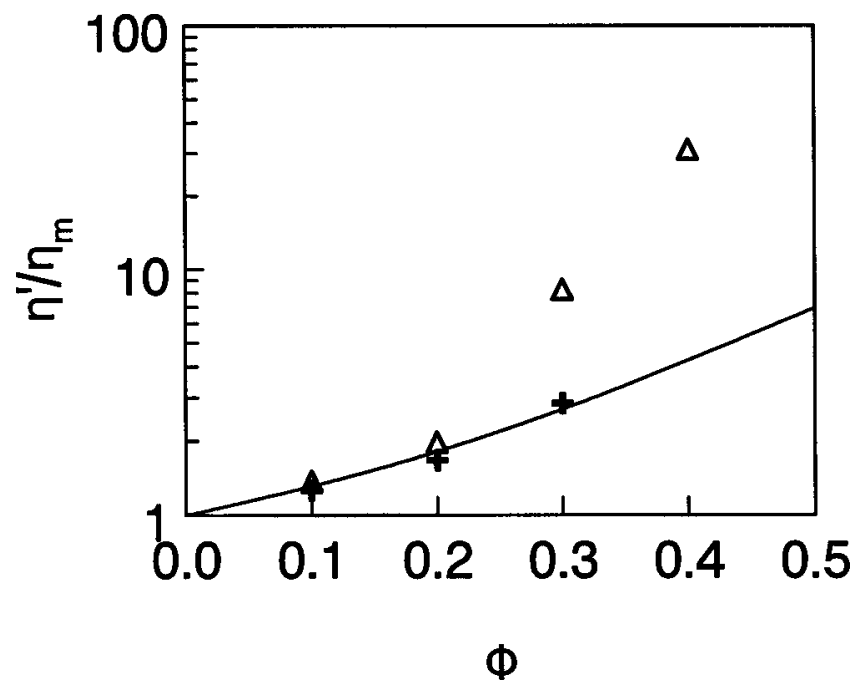

FIG. 9. Volume fraction dependence of $\eta^{\prime} / \eta_{m}(\omega=0)$ at low frequencies for suspensions of spheres in viscoelastic media; $\triangle, \boldsymbol{\Lambda}$ : data of Aral and Kaylon (1997); +: data of Chan and Powell (1984); solid line: 1 $+\eta_{H} / \eta_{m}(\omega=0)$, calculated using Eq. (38). 
TABLE II. Fit parameters for the Maxwell modes of the suspending medium, used in the analysis of the experiments of Aral and Kaylon (1997); the Brownian time for this system is given by $t_{B}=2.3$ $\times 10^{-11} \mathrm{~s}$

\begin{tabular}{cccc}
\hline \hline$k$ & $\begin{array}{c}\eta_{k} \\
\left(0^{3} \tilde{\tau}_{k}\right. \\
(\mathrm{s})\end{array}$ & $10^{10} \tau_{k}$ \\
\hline 1 & 43.3 & 2.93 & 5.31 \\
2 & 21.2 & 0.386 & 0.70 \\
3 & 31.7 & 20.17 & 36.57 \\
\hline \hline
\end{tabular}

is found to be frequency thinning for $\omega>10^{8}$. In Fig. 10 we show the data of Aral and Kaylon, divided by the prediction of Eq. (42), using the values of $\eta_{k}$ and $\tau_{k}$ given in Table II. For $G^{\prime \prime}$ we find good agreement between theory and experiment. The results for $G^{\prime}$ are reproduced well by the theory for $\omega>10^{8}$. For smaller frequencies we find that the theory underestimates $G^{\prime}$. However, at frequencies of $\omega<10^{8}$ the values of $G^{\prime}$ are roughly an order of magnitude smaller than those of $G^{\prime \prime}$. This may hamper accurate determination of $G^{\prime}$.

Chan and Powell (1984) used a similar approach to interpret their results; they used experimental data for spheres in a viscous medium instead of our Eq. (38). They also found fair agreement between theory and experiment. Our results support their analysis. However, our results also show clearly that their analysis ceases to be valid for frequencies at which the Brownian motion of the particles contributes significantly to the bulk stress. An example of this is presented in Fig. 8.

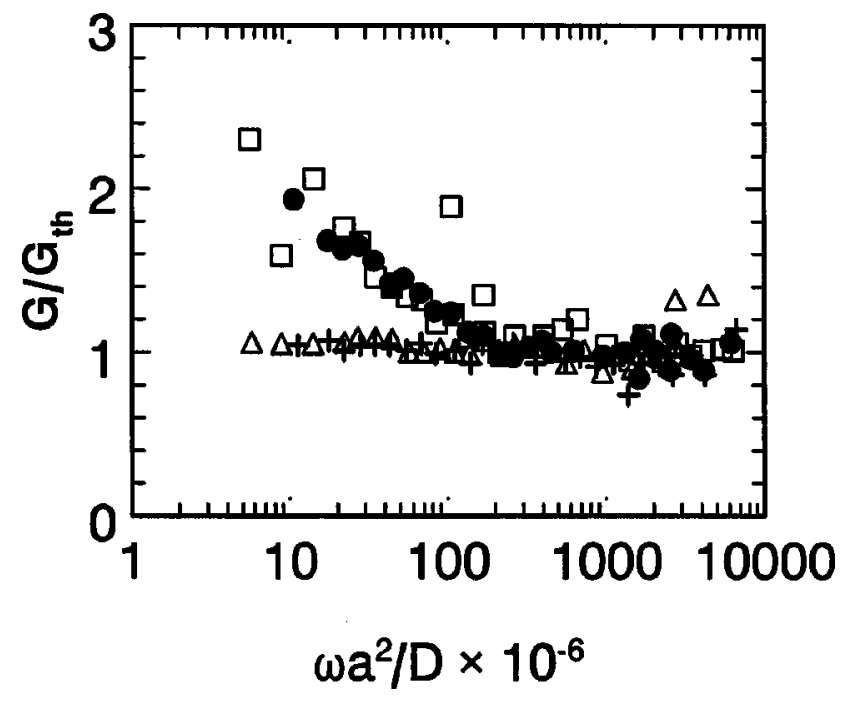

FIG. 10. Frequency dependence of the ratio $G^{\prime \prime}$ (experiment) $/ G^{\prime \prime}$ (theory) for $\phi=0.1(+)$ and $\phi=0.2(\triangle)$, and the ratio $G^{\prime}$ (experiment) $/ G^{\prime}$ (theory) for $\phi=0.1(\bullet)$ and $\phi=0.2(\square)$; the experimental data for spheres suspended in a viscoelastic medium are taken from Aral and Kaylon (1997); the theoretical predictions are based on Eqs. (42) and (38) in combination with the values given in Table II. 


\section{CONCLUSIONS}

On the basis of the correspondence principle we have developed in this article an extension of the Stokesian dynamics method in order to be able to study the rheology of systems of spheres suspended in a viscoelastic Maxwell medium. It turns out that the dynamics of spheres suspended in a Maxwell medium, characterized by one relaxation time, are identical to those of spheres suspended in a viscous medium. This is a consequence of the fact that, on the time scale of interest $t_{B} \ll t \leqslant \tau$, particle momentum relaxation and particle inertia can be neglected. As a consequence, the contribution of Brownian motion to the bulk stress is found to be identical to the corresponding contribution in the viscous case. Our simulation results for suspensions with a viscous matrix suggest that it is justified to neglect inertial effects for the range of frequencies considered here.

We have performed Stokesian dynamics simulations for suspensions of spheres in a viscous medium as well as of spheres in a Maxwell medium. Systems of spheres suspended in a viscous medium were studied for two reasons. The first reason is that up to now no quantitative comparison was made between available experimental results and computer simulations of suspensions subjected to an oscillatory strain. Second, we expected these results to be helpful in the interpretation of results obtained in our simulations of systems of spheres suspended in a Maxwell medium. The most striking result of our simulations of systems of spheres suspended in a viscous medium is the observation that the frequency dependence of the elastic modulus $G^{\prime}$ attains a plateau value for high frequencies. Such a plateau was not observed in the experiments of van der Werff et al. (1989), whereas the experiments of Shikata and Pearson (1994) did reveal a plateau for $G^{\prime}$. Our simulations of systems of spheres suspended in a Maxwell medium have shown that it is possible to accurately predict the hydrodynamic contribution to the complex viscosity on the basis of data for suspension with a viscous matrix in combination with use of Eqs. (38) and (39). We have applied these equations successfully to the experimental data for $\phi=0.1$ and 0.2 of Aral and Kaylon (1997). These experiments were done at frequencies that are inaccessible for Stokesian dynamics simulations.

\section{ACKNOWLEDGMENTS}

The authors like to thank Professor J. F. Brady (CalTech) for kindly providing the Stokesian dynamics code written by T. H. Phung, and for the pleasant discussions at the start of this project. They also would like to thank Professor W. J. Briels for the opportunity he gave them to do some of their simulations on the HPC computer. Finally they would like to thank J. Padding for his assistance in the startup phase on this computer. This work was financially supported by DSM Research (Geleen, The Netherlands).

\section{APPENDIX}

In the Appendix we will indicate how the Langevin equation in configuration space, Eq. (32), i.e.,

$$
\frac{d r}{d t}(t)=v^{\infty}(t)+\frac{k_{B} T}{\eta_{m}} \nabla\left[R_{f v}(t)\right]^{-1}+\left[R_{f v}(t)\right]^{-1}\left(R_{f D}(t) D^{\infty}(t)+\sqrt{\frac{k_{B} T}{\eta_{m}}} g(t) \xi(t)\right),
$$

can be derived from the Langevin equation in the extended phase $(r, v, a)$ space, Eq. (31), i.e., 


$$
\begin{gathered}
\frac{d r}{d t}(t)=v(t), \quad \frac{d v}{d t}(t)=a(t), \\
\tau \frac{d a}{d t}(t)=-a(t)+\frac{\eta_{m}}{m}\left\{R_{f v}\left[v^{\infty}(t)-v(t)\right]+R_{f D}(t) D^{\infty}(t)\right\}+\frac{\sqrt{k_{B} T \eta_{m}}}{m} g(t) \xi(t),
\end{gathered}
$$

by a method called adiabatic elimination. This well known technique from nonequilibrium statistical mechanics [see, for instance, Haken (1978)] involves elimination of "fast" or irrelevant degrees of freedom from the description of a system on a "slow" or relevant time scale of interest, resulting in a reduced and simpler description in terms of the remaining slow degrees of freedom. This contraction of the dynamics rests upon the idea that on the slow time scale of interest these fast variables have already attained their equilibrium values.

The generic example of adiabatic elimination is the transition from a description in terms of the position and momentum of a single Brownian particle to a description which only involves its position [see, for instance, Gardiner (1985)]. In that case adiabatic elimination is straightforward and one obtains the correct reduced description in the high-friction limit, i.e., the Langevin equation for the position of the particle, by simply setting the time derivative of the momentum (the fast variable) equal to zero in the phase-space equation of motion. However, one has to realize that this Langevin equation for the position of the particle is in fact a zeroth-order approximation, valid for infinite friction. For finite friction there are corrections to this reduced description which account for the finite relaxation time of the fast variable. The same straightforward procedure can also be applied to systems consisting of a number of Brownian particles as long as these particles do not interact hydrodynamically (the free draining case). When hydrodynamic interactions are present, one can no longer use the above naive approach [Sancho, San Miquel and Dürr (1982)]. Using that approach, for instance, would lead to contracted dynamics which do not show the proper equilibrium behavior (the correct Boltzmann distribution for the slow variables) for $t \rightarrow \infty$. The reason for the naive approach breaking down in this case can be found in the nonunique interpretation of the resulting Langevin equation in configuration space after the momenta have been eliminated [Hasegawa, Mabuchi, and Baba (1980)]. Instead, one has to use an alternative, rather subtle, elimination procedure [Sancho, San Miquel, and Dürr (1982)]. In the remainder of this article this procedure will be used to show how not only the velocities but also the accelerations of the Brownian particles can be eliminated from the full dynamics described by Eq. (A2). This will then result in the contracted dynamics as given by Eq. (A1). In order to simplify the presentation without sacrificing the essence of the method, we will follow Sancho, San Miquel, and Dürr (1982) by considering the physically analogous, but mathematically simpler, problem of a one-dimensional Brownian particle in an inhomogeneous viscoelastic medium. In the higher-dimensional case, i.e., Eq. (A2), the same line of reasoning and the same steps need to be taken as in the one-dimensional problem and so the final result, i.e., Eq. (A1), can simply be obtained by redoing the analysis step by step. The approach taken is necessarily more physical than mathematical in nature because rigorous methods of analysis are not available at present for the kind of problems we are confronted with here. Consider therefore the following set of equations of motion describing a one-dimensional Brownian particle immersed in a linear viscoelastic inhomogeneous Maxwell medium with one relaxation time $\tau$ at a temperature $T$ :

$$
\dot{x}(t)=v(t), \quad \dot{v}(t)=a(t)
$$




$$
\tau \dot{a}(t)=-a(t)-\lambda[x(t)] v(t)-\phi[x(t)]+\mu[x(t)] \xi(t),
$$

with $\xi(t)$ a Gaussian white noise process obeying

$$
\begin{gathered}
\langle\xi(t)\rangle \equiv 0, \\
\left\langle\xi(t) \xi\left(t^{\prime}\right)\right\rangle \equiv 2 \delta\left(t-t^{\prime}\right) .
\end{gathered}
$$

The inhomogeneous nature of the medium reveals itself through the fact that in addition to the presence of the $\phi(x)$ term, which is the analog of the term $\left(\eta_{m} / m\right)\left[R_{f v} v^{\infty}(t)\right.$ $\left.+R_{f D}(t) D^{\infty}(t)\right]$ in Eq. (A2), both the friction coefficient $\lambda(x)$ and the noise strength $\mu(x)$ in the equation for the acceleration are position dependent. Both these quantities are related in the following way by the fluctuation dissipation theorem:

$$
\mu^{2}(x)=k_{B} T \lambda(x)
$$

We will assume that the friction coefficient $\lambda(x)$ is large in the sense that we will take $\lambda(x) \equiv \lambda_{0}+\delta \lambda(x)$ with $\lambda_{0} \gg 1$ and $0 \leqslant \delta \lambda(x) \ll \lambda_{0}$. When $\tau=0$ Eq. (A3) reduces to the problem that was studied by Sancho, San Miquel, and Dürr (1982).

It is clear that Eq. (A3) contains two distinct time scales, the Brownian time scale which is of the order of $1 / \lambda_{0}$ and the viscoelastic time scale $\tau$ which is supposed to be much larger than this Brownian time scale. Because we are interested in the influence of the viscoelasticity of the medium on the dynamics of the Brownian particle we will consider Eq. (A3) for times $t$ in the regime in between, i.e., for $1 / \lambda_{0} \ll t \leqslant \tau$. In this regime the so-called elasticity number is very large, i.e., $\tau / t_{B} \equiv \lambda_{0} \tau \gg 1$. Furthermore, it also follows that in this regime the inverse of the geometric mean of $1 / \lambda_{0}$ and $\tau$ is very large, i.e., $\sqrt{\lambda_{0} / \tau} \gg 1$, a result which we will use below. Thus, after substituting the second equation of Eq. (A3) into the third one and dividing by $\tau$, we end up with

$$
\ddot{v}(t)+\frac{1}{\tau} \dot{v}(t)+\frac{\lambda[x(t)]}{\tau} v(t)=\frac{1}{\tau}\{-\phi[x(t)]+\mu[x(t)] \xi(t)\} .
$$

Adiabatic elimination of both the velocity $v(t)$ and the acceleration $a(t)$ from Eq. (A3) in essence comes down to solving this second-order Langevin equation with multiplicative noise, Eq. (A6), for $v(t) \equiv(d x / d t)(t)$ in terms of $x(t)$ and $\xi(t)$. In general, one can only solve Langevin equations in a formal sense, say, in the form of a quadrature involving the noise term. In their analysis of Eq. (A3) for $\tau=0$, Sancho, San Miquel, and Dürr took such a formal solution as the starting point in the derivation of the correct Langevin equation for $x(t)$. Unfortunately, due to the time dependence of the friction coefficient, it is not possible to write a formal solution of Eq. (A6). However, with

$$
\int_{t_{0}}^{t} d s \lambda[x(s)] \simeq \lambda_{0}\left(t-t_{0}\right) \simeq \lambda[x(t)]\left(t-t_{0}\right)
$$

due to our assumptions concerning $\lambda[x(t)]$, it follows that an approximate solution of Eq. (A6), after the transients involving the initial velocity and the initial acceleration have decayed, is given by 


$$
\begin{aligned}
v(t) \simeq & -\frac{1}{\tau} \int_{-\infty}^{t} d s \exp \left(-\frac{t-s}{\tau}\right)\left(\sqrt{\frac{\tau}{\lambda(x(t))}} \sin \left[\sqrt{\frac{\lambda[x(t)]}{\tau}}(t-s)\right]\right) \phi[x(s)] \\
& +\frac{1}{\tau} \int_{-\infty}^{t} d s \exp \left(-\frac{t-s}{\tau}\right)\left[\sqrt{\frac{\tau}{\lambda[x(t)]}} \sin \left(\sqrt{\frac{\lambda[x(t)]}{\tau}}(t-s)\right)\right] \mu[x(s)] \xi(s) .
\end{aligned}
$$

Taking the lower limit $t_{0}$ in these integrals to be $-\infty$ is just a rigorous and formal procedure to enforce these transients to be absent. Now, a further approximation to Eq. (A8) can be obtained by noting that the sine function is a rapidly varying function of $s$ for large $\sqrt{\lambda[x(t)] / \tau}$. Therefore the major contribution to each of the time integrals will come from its integrand evaluated at the endpoint $s=t$. Mathematically, this is equivalent to making use of the following approximation, valid for large $\sqrt{\lambda[x(t)] / \tau}$ :

$$
\sqrt{\frac{\tau}{\lambda[x(t)]}} \sin \left(\sqrt{\frac{\lambda[x(t)]}{\tau}}(t-s)\right) \simeq \frac{2 \tau}{\lambda[x(t)]} \delta(t-s),
$$

which, in fact, is the first term in a formal expansion of the left-hand side in powers of $1 / \lambda[x(t)]$. This leads to

$$
v(t)=-\frac{\phi[x(t)]}{\lambda[x(t)]}+\frac{\mu[x(t)]}{\lambda[x(t)]} \xi(t)+\ldots,
$$

where, on account of Eq. (A5), it follows that these two contributions to $v(t)$ are, respectively, of order $\lambda_{0}^{-1}$ and $\lambda_{0}^{-1 / 2}$. Equation (A10) is not a consistent approximation of Eq. (A8) to order $\lambda_{0}^{-3 / 2}$, because there is an additional contribution of order $\lambda_{0}^{-1}$ missing in Eq. (A10). This term can be determined in the following way. First we integrate Eq. (A10) to obtain

$$
x(s) \simeq x(t)+\int_{s}^{t} d t^{\prime} \phi\left[x\left(t^{\prime}\right)\right] \lambda\left[x\left(t^{\prime}\right)\right]-\int_{s}^{t} d t^{\prime} \frac{\mu\left[x\left(t^{\prime}\right)\right]}{\lambda\left[x\left(t^{\prime}\right)\right]} \xi\left(t^{\prime}\right),
$$

which allows us to write

$$
\begin{aligned}
\mu[x(s)] & \simeq \mu[x(t)]+\frac{d \mu[x(t)]}{d x(t)}[x(s)-x(t)] \\
& =\mu[x(t)]-\frac{d \mu[x(t)]}{d x(t)} \mu[x(t)] \int_{s}^{t} d t^{\prime} \frac{\xi\left(t^{\prime}\right)}{\lambda\left[x\left(t^{\prime}\right)\right]}+\mathcal{O}\left(\lambda_{0}^{-1 / 2}\right),
\end{aligned}
$$

and similarly,

$$
\phi[x(s)]=\phi[x(t)]+\mathcal{O}\left(\lambda_{0}^{-1 / 2}\right) .
$$

If we now substitute these last two results into Eq. (A8) and use Eq. (A9), we end up with the following consistent improvement to Eq. (A10):

$$
v(t)=\dot{x}(t)=-\frac{\phi[x(t)]}{\lambda[x(t)]}+\frac{\mu[x(t)]}{\lambda[x(t)]} \xi(t)-\frac{1}{\tau} \frac{d \mu[x(t)]}{d x(t)} \mu[x(t)] \int_{-\infty}^{t} d s \xi(s) \exp \left(-\frac{t-s}{\tau}\right)
$$




$$
\times\left[\sqrt{\frac{\tau}{\lambda[x(t)]}} \sin \left(\sqrt{\frac{\lambda[x(t)]}{\tau}}(t-s)\right)\right] \int_{s}^{t} d t^{\prime} \frac{\xi\left(t^{\prime}\right)}{\lambda\left[x\left(t^{\prime}\right)\right]}+\mathcal{O}\left(\lambda_{0}^{-3 / 2}\right) .
$$

We did not use Eq. (A9) to simplify the third contribution in this expression because this essentially Markovian approximation would cause it to vanish altogether. Instead, it can be simplified by considering the associated Smoluchowski equation for the probability density $P(x, t)$ [see, for instance, van Kampen (1981)]. This approximate equation, due to the non-Markovian nature of Eq. (A14), can be constructed from the stochastic Liouville equation or continuity equation in configuration space for the density $\rho(x, t)$ describing an ensemble of points obeying Eq. (A14), i.e.,

$$
\frac{\partial \rho(x, t)}{\partial t}=-\frac{\partial}{\partial x}[\dot{x}(t) \rho(x, t)] .
$$

If we average this equation over the white noise $\xi(t)$, we obtain the following equation for this probability density $P(x, t) \equiv\langle\rho(x, t)\rangle$ :

$$
\begin{aligned}
\frac{\partial P(x, t)}{\partial t}= & \frac{\partial}{\partial x}\left(\frac{\phi(x)}{\lambda(x)} P(x, t)\right)-\frac{\partial}{\partial x}\left(\frac{\mu(x)}{\lambda(x)}\langle\xi(t) \rho(x, t)\rangle\right)+\frac{\partial}{\partial x}\left\{\frac{d \mu(x)}{d x} \mu(x) \frac{1}{\tau} \int_{-\infty}^{t} d s \exp \left(-\frac{t-s}{\tau}\right)\right. \\
& \left.\times\left[\sqrt{\frac{\tau}{\lambda(x)}} \sin \left(\sqrt{\frac{\lambda(x)}{\tau}}(t-s)\right)\right] \int_{s}^{t} d t^{\prime} \frac{1}{\lambda(x)}\left\langle\xi(s) \xi\left(t^{\prime}\right) \rho(x, t)\right\rangle\right\} .
\end{aligned}
$$

To complete this equation we need to evaluate the white noise averages $\langle\xi(t) \rho(x, t)\rangle$ and $\left\langle\xi(s) \xi\left(t^{\prime}\right) \rho(x, t)\right\rangle$. This can be done by invoking the well known Novikov theorem [Novikov (1965)]. To the same level of approximation as that used in Eq. (A14) this leads to

$$
\langle\xi(t) \rho(x, t)\rangle \simeq-\frac{\partial}{\partial x}[\mu(x) \lambda(x) P(x, t)],
$$

and

$$
\left\langle\xi(s) \xi\left(t^{\prime}\right) \rho(x, t)\right\rangle \simeq 2 \delta\left(s-t^{\prime}\right) P(x, t) .
$$

By substituting these last two expressions into Eq. (A16) and using Eqs. (A9) and (A5), the Smoluchowski equation we are looking for results in

$$
\begin{aligned}
\frac{\partial P(x, t)}{\partial t} & =\frac{\partial}{\partial x}\left(\frac{\phi(x)}{\lambda(x)} P(x, t)\right)+\frac{\partial}{\partial x}\left[\frac{\mu(x)}{\lambda(x)} \frac{\partial}{\partial x}\left(\frac{\mu(x)}{\lambda(x)} P(x, t)\right)\right]+\frac{\partial}{\partial x}\left(\frac{\mu(x)}{\lambda(x)^{2}} \frac{d \mu(x)}{d x} P(x, t)\right) \\
& =\frac{\partial}{\partial x}\left[\frac{1}{\lambda(x)}\left(\phi(x)+k_{B} T \frac{\partial}{\partial x}\right) P(x, t)\right] .
\end{aligned}
$$

In the case where $\phi(x)$ corresponds to the derivative of some potential $U(x)$ i.e., $\phi(x)=U^{\prime}(x)$, Eq. (A19) leads to the correct equilibrium distribution (Boltzmann distribution) $P_{\mathrm{eq}}(x) \sim \exp \left\{-\left[U(x) / k_{B} T\right]\right\}$. This Smoluchowski equation is stochastically equivalent to the following Langevin equation in the Itô interpretation [van Kampen (1981)]: 


$$
\frac{d x}{d t}=-\frac{\phi(x)}{\lambda(x)}-\frac{2 \mu(x)}{\lambda(x)^{2}} \frac{d \mu(x)}{d x}+\frac{\mu(x)}{\lambda(x)} \xi(t)=-\frac{\phi(x)}{\lambda(x)}+k_{B} T \frac{d}{d x}\left(\frac{1}{\lambda(x)}\right)+\sqrt{\frac{k_{B} T}{\lambda(x)}} \xi(t) .
$$

It is easy to see that this result corresponds to Eq. (A14) in which the term involving the two $\xi$ 's was averaged. Equation (A20) is now the correct reduced description for the slow variable $x$ when the fast variables $v$ and $a$ have been eliminated from Eq. (A3). As was mentioned before the analysis of the higher-dimensional case proceeds along the same lines of reasoning: clearly Eq. (A20) has the same structure as Eq. (A1), in the same way that Eq. (A3) resembles Eq. (A2). An analogous result for the case of a single Brownian particle in a Maxwell fluid was obtained by Volkov and Leonov (1996).

\section{References}

Aral, B. K. and D. M. Kalyon, "Viscoelastic material functions of noncolloidal suspensions with spherical particles,'” J. Rheol. 41, 599-620 (1997).

Batchelor, G. K., "The stress system in a suspension of force-free particles," J. Fluid Mech. 41, 545-570 (1970).

Batchelor, G. K., "The effect of Brownian motion on the bulk stress in a suspension of spherical particles," J. Fluid Mech. 83, 97-117 (1977).

Batchelor, G. K. and J. T. Green, "The determination of the bulk stress in a suspension of spherical particles to the order $c^{2}$," J. Fluid Mech. 56, 401-427 (1972).

Boek, E. S., P. V. Coveney, H. N. W. Lekkerkerker, and P. van der Schoot, "Simulating the rheology of dense colloidal suspensions using dissipative particle dynamics," Phys. Rev. E 55, 3124-3133 (1997).

Bossis, G. and J. F. Brady, "Dynamic simulation of sheared suspensions. I. General method," J. Chem. Phys. 80, 5141-5154 (1984).

Bossis, G. and J. F. Brady, “The rheology of Brownian suspensions,' J. Chem. Phys. 91, 1866-1874 (1989).

Brady, J. F., "The rheological behavior of concentrated colloidal dispersions," J. Chem. Phys. 99, 567-581 (1993).

Brady, J. F. and G. Bossis, "Stokesian Dynamics," Annu. Rev. Fluid Mech. 20, 111-157 (1988).

Brenner, H., "The Stokes resistance of an arbitrary particle. IV. Arbitrary fields of flow," Chem. Eng. Sci. 19, 703-727 (1964).

Chan, D. and R. L. Powell, "Rheology of suspensions of spherical particles in a Newtonian and a nonNewtonian fluid," J. Non-Newtonian Fluid Mech. 15, 165-179 (1984).

Christensen, R. M., Theory of Viscoelasticity, An Introduction (Academic, New York, 1971).

Feng, J., P. Y. Huang, and D. D. Joseph, "Dynamic simulation of solid particles in an Oldroyd-B fluid," J. Non-Newtonian Fluid Mech. 63, 63-88 (1996).

Gardiner, C. W., Handbook of Stochastic Methods (Springer, Berlin, 1985).

Giraud, L., D. d'Humieres, and P. Lallemand, "A lattice Boltzmann model for Jeffery's viscoelastic fluid," Europhys. Lett. 42, 625-630 (1998)

Haken, H., Synergetics, An Introduction, Springer Series in Synergetics Vol. 1, 2nd ed. (Springer, Berlin, 1978).

Hasegawa, H., M. Mabuchi, and T. Baba, "On the usefulness of stochastic calculus applied to adiabatic elimination in Langevin equations,"' Phys. Lett. 79A, 273-277 (1980).

Heyes, D. M., "Molecular simulations of colloidal liquids," J. Colloid Interface Sci. 51, 247-268 (1994).

Heyes, D. M. and P. J. Mitchell, "Linear viscoelasticity of concentrated hard sphere dispersions," J. Phys.: Condens. Matter 6, 6423-6436 (1994).

Huang, P. Y., H. H. Hu, and D. D. Joseph, "Direct simulation of the sedimentation of elliptic particles in Oldroyd-B fluids," J. Fluid Mech. 362, 297-325 (1998).

Jongschaap, R. J. J. and J. Mellema, “Stress tensor expressions for dispersions,"’ J. Rheol. 39, 953-959 (1995).

Kim S. and S. J. Karrila, Microhydrodynamics, Principles and Selected Applications (Butterworth-Heinemann, Boston, 1991).

Koelman, J. M. V. A. and P. J. Hoogebrugge, "Dynamic simulations of hard-sphere suspensions under steady shear,' Europhys. Lett. 21, 363-368 (1993).

Kubo, R., M. Toda, and N. Hashitsume, Statistical Physics II (Springer, Berlin, 1991).

Ladd, A. J. C., "Hydrodynamic transport coefficients of random dispersions," J. Chem. Phys. 93, 3484-3494 (1990).

Ladd, A. J. C., "Numerical simulations of particulate suspensions via a discretized Boltzmann equation. Part 2. Numerical results," J. Fluid Mech. 271, 311-339 (1994). 
Lionberger, R. A. and W. B. Russel, “High frequency modulus of hard sphere colloids,' J. Rheol. 38, 18851908 (1994).

Macosko, C. W., Rheology, Principles Measurements and Applications (VCH-Wiley, New York, 1994).

Mellema, J., C. G. de Kruif, C. Blom, and A. Vrij, "Hard sphere colloidal dispersions: Mechanical relaxation pertaining to thermodynamic forces," Rheol. Acta 26, 40-44 (1987).

Nägele, G., "'On the dynamics and structure of charge-stabilized suspensions,'” Phys. Rep. 272, $215-372$ (1996).

Novikov, E. A., “'Functionals and random force method in turbulence theory,', Sov. Phys. JETP 20, 1290-1304 (1965).

Ohl, N. and W. Gleissle, "The characterization of the steady-state and normal stress functions of highly concentrated suspensions formulated with viscoelastic fluids,' J. Rheol. 37, 381-406 (1992).

Phillips, R. J., "Dynamic simulation of hydrodynamically interacting spheres in a quiescent second order fluid,’’ J. Fluid Mech. 315, 325-365 (1996).

Phung, T. H., Ph.D. thesis, California Institute of Technology, 1993.

Phung, T. H., J. F. Brady, and G. Bossis, "Stokesian dynamics of Brownian suspensions,' J. Fluid Mech. 313, 181-207 (1996).

Sancho, J. M., M. San Miguel, and D. Dürr, “Adiabatic elimination for systems of Brownian particles with nonconstant damping coefficients," J. Stat. Phys. 28, 291-305 (1982).

Schnurr, B., F. Gittes, F. C. MacKintosh, and C. F. Schmidt, "Determining microscopic viscoelasticity in flexible and semiflexible polymer networks from thermal fluctuations,', Macromolecules 30, 7781-7796 (1997).

Shikata, T. and D. S. Pearson, "Viscoelastic behavior of concentrated spherical suspensions," J. Rheol. 38, 601-616 (1994).

Toivakka, M., D. Eklund, and D. W. Bousfield, "Prediction of suspension viscoelasticity through particle motion modeling,', J. Non-Newtonian Fluid Mech. 56, 49-64 (1995).

Tough, R. J. A., P. N. Pusey, H. N. W. Lekkerkerker, and C. van den Broeck, "Stochastic descriptions of the dynamics of interacting Brownian particles,'” Mol. Phys. 59, 595-619 (1987).

van Kampen, N. G., Stochastic Processes in Physics and Chemistry (North-Holland, Amsterdam, 1981).

van der Werff, J. C., C. G. de Kruif, C. Blom, and J. Mellema, "Linear viscoelastic behavior of dense hard-sphere dispersions,"' Phys. Rev. A 39, 795-807 (1989).

Verberg, R., I. M. de Schepper, and E. G. D. Cohen, “Viscosity of colloidal suspensions,' Phys. Rev. E 55, 3143-3158 (1997).

Volkov, V. S. and A. I. Leonov, "Non-Markovian Brownian motion in a viscoelastic fluid," J. Chem. Phys. 104, 5922-5931 (1996).

Volkov, V. S. and G. V. Vinogradov, "Theory of dilute polymer solutions in viscoelastic fluid with a single relaxation time,' J. Non-Newtonian Fluid Mech. 15, 29-44 (1984).

Wagner, A. J. L. Giraud, and C. E. Scott, "Simulation of a cusped bubble rising in a viscoelastic fluid with a new numerical method,' preprint in xxx.lanl.gov cond matter/9904029.

Woutersen, A. T. J. M., J. Mellema, C. Blom, and C. G. de Kruif, "Linear viscoelasticity in dispersions of adhesive hard spheres,' J. Chem. Phys. 101, 542-553 (1994). 